\title{
ON LIFTING FROM CLASSICAL GROUPS TO GL
}

\author{
by J. W. GOGDELL, H. H. KIM, I. I. PIATETSKI-SHAPIRO and F. SHAHIDI
}

One of the most central questions in the theory of automorphic forms is that of Langlands' functoriality or the lifting of automorphic representations [20, 2]. One of the most basic cases of functoriality would be the lifting of automorphic representations from the split classical groups to an appropriate $\mathrm{GL}_{\mathrm{N}}$. It is this question we address in this paper.

Take $\mathrm{G}$ to be a split classical group over a number field $k$. So $\mathrm{G}$ is one of the groups $\mathrm{SO}_{2 n+1}, \mathrm{SO}_{2 n}$, or $\mathrm{Sp}_{2 n}$. The connected component ${ }^{\mathrm{L}} \mathrm{G}^{0}$ of the Langlands dual group is $\mathrm{Sp}_{2 n}(\mathbf{C}), \mathrm{SO}_{2 n}(\mathbf{C})$, or $\mathrm{SO}_{2 n+1}(\mathbf{C})$ respectively. In each case there is a natural embedding of ${ }^{\mathrm{L}} \mathrm{G}^{0}$ into $\mathrm{GL}_{\mathrm{N}}(\mathbf{C})={ }^{\mathrm{L}} \mathrm{GL}_{\mathrm{N}}^{0}$ for $\mathrm{N}=2 n, 2 n$, or $2 n+1$ respectively. The philosophy of Langlands then says that associated to this map of dual groups there should exist a lifting of automorphic forms on $\mathrm{G}\left(\mathbf{A}_{k}\right)$ to $\mathrm{GL}_{\mathrm{N}}\left(\mathbf{A}_{k}\right)$. This map on dual groups also governs local liftings of irreducible admissible representations of $\mathrm{G}\left(k_{v}\right)$ to $\mathrm{GL}_{\mathrm{N}}\left(k_{v}\right)$ and these local and global liftings should be compatible.

While there is at present no precise global conjecture on the nature of the lifting in our situation, the local lifting is well understood in several cases and the global lifting can be understood in terms of compatibility with the local lifts. If $v$ is an archimedean place of $k$ then every irreducible admissible representation $\pi_{v}$ of $\mathrm{G}\left(k_{v}\right)$ is given by an admissible homomorphism of the local Weil group $\mathrm{W}_{k_{v}}$ into ${ }^{\mathrm{L}} \mathrm{G}^{0}[23,2]$. Composing with the map to ${ }^{\mathrm{L}} \mathrm{GL}_{\mathrm{N}}^{0}$ we get a parameter for an irreducible admissible representation $\Pi_{v}$ of $\mathrm{GL}_{\mathrm{N}}\left(k_{v}\right) . \Pi_{v}$ is the local Langlands lift of $\pi_{v}$. Similarly, if $v$ is a non-archimedean place and $\pi_{v}$ an unramified admissible representation then $\pi_{v}$ is determined by its Satake parameter $\left[t_{v}\right]$ which is a semi-simple conjugacy class in ${ }^{\mathrm{L}} \mathrm{G}^{0}[29,2]$. The image of $\left[t_{v}\right]$ under the L-homomorphism determines a conjugacy class in ${ }^{\mathrm{L}} \mathrm{GL}_{\mathrm{N}}^{0}$ and hence Satake parameters for an unramified representation $\Pi_{v}$ of $\mathrm{GL}_{\mathrm{N}}\left(k_{v}\right)$. Again, $\Pi_{v}$ is the local Langlands lift of $\pi_{v}$.

If we have an irreducible automorphic representation $\pi=\otimes^{\prime} \pi_{v}$ of $\mathrm{G}\left(\mathbf{A}_{k}\right)$ then for almost all places, namely the archimedean ones and the non-archimedean places where $\boldsymbol{\pi}_{v}$ is unramified, the local component $\boldsymbol{\pi}_{v}$ of $\pi$ has a local lift $\Pi_{v}$. We will say that a automorphic representation $\Pi=\otimes^{\prime} \Pi_{v}$ of $\mathrm{GL}_{\mathrm{N}}\left(\mathbf{A}_{k}\right)$ is a weak Langlands lift of $\pi$, or simply a weak lift, if at the archimedean places and almost all non-archimedean places where $\pi_{v}$ is unramified $\Pi_{v}$ is in fact the local lift of $\pi_{v}$.

J. C. was supported in part by the NSA. H. K., I. P. S., and F. S. were supported in part by the NSF. While at the Institute for Advanced Study, J. C. was supported in part by a grant from the Ellentuck Fund, H. K. from NSF grant DMS 9729992, and I. P. S. from the general funds of the Institute. The final stage of the research by J. C. and I. P. S. was conducted for the Clay Mathematics Institute. 
In this paper we establish the existence of a weak lift in this sense of globally generic cuspidal representations of $\mathrm{SO}_{2 n+1}\left(\mathbf{A}_{k}\right)$ to $\mathrm{GL}_{2 n}\left(\mathbf{A}_{k}\right)$.

There are currently three methods of establishing functoriality. Two of them are trace formula methods: the usual trace formula of Arthur and the relative trace formula of Jacquet. The third method is that of converse theorems [8]. It is this third method which we have developed with this purpose in mind and which we use to establish these lifts.

The converse theorem is a method of L-functions. There are currently two ways to analyze the analytic properties of L-functions: through integral representations or through the constant term and Fourier coefficients of Eisenstein series. This work is a blending of these two methods. The converse theorem itself is from the theory of integral representations, for in its essence it is the inversion of the integral representation of L-functions for $\mathrm{GL}_{\mathrm{N}}$. To effect the lift from a classical group $\mathrm{G}$ using the converse theorem we must have relatively complete control of the analytic properties of the Lfunctions for G. In this paper we do this almost entirely using the method of Eisenstein series. The results we use which rely on this method are available for all split classical groups. The one exception is the local "stability of $\gamma$ " (Proposition 3.4) which lets us finesse the local lifting at the ramified places. This is a result from the local theory of integral representations and is currently only available for $\mathrm{SO}_{2 n+1}$. Once this result is established for $\mathrm{SO}_{2 n}$ or $\mathrm{Sp}_{2 n}$, the lifting from these groups will follow.

The first section of the paper is devoted to the precise statement of the lifting for the case of $\mathrm{G}=\mathrm{SO}_{2 n+1}$. The second section contains a discussion of the version of the converse theorem we utilize. The third section is devoted to the analytic properties of L-functions for $\mathrm{SO}_{2 n+1}$. In the fourth section we discuss the local liftings at all places and how the stability of $\gamma$ lets us finesse the local lifting at the finite places where $\pi_{v}$ is ramified. In the fifth section we effect the lifting. The sixth section is devoted to consequences of the lift, including the existence of a local lift for the case of supercuspidal representations.

We would like to thank our colleagues that have supported us in this project over the years, most particularly Steve Gelbart, David Ginzburg, Steve Rallis, and David Soudry. We also thank the Institute for Advanced Study for bringing us together as part of their Special Year in the Theory of Automorphic Forms and L-functions, and particularly the organizers of the special year E. Bombieri, H. Iwaniec, R. P. Langlands, and P. Sarnak, for it was there that we finally brought all the pieces together.

\section{Lifting from $\mathrm{SO}_{2 n+1}$ to $\mathrm{GL}_{2 n}$}

Let $k$ be a number field and let $\mathbf{A}=\mathbf{A}_{k}$ be its ring of adeles. We fix a non-trivial continuous additive character $\psi$ of $\mathbf{A}$ which is trivial on the principal adeles $k$.

Let $\mathrm{G}_{n}=\mathrm{SO}_{2 n+1}$ be the split special orthogonal group in $2 n+1$ variables defined over $k$. For definiteness, we will take $\mathrm{G}_{n}$ as the isometry group of the form 
$\Phi=\left(\begin{array}{lll} & . & \\ 1 & & \end{array}\right)$. In this realization we can take the standard Borel subgroup of $\mathrm{G}_{n}$ to be represented by upper triangular matrices. We will denote this Borel subgroup by $\mathrm{B}_{n}$ and its unipotent radical by $\mathrm{U}_{n}$. The abelianization of $\mathrm{U}_{n}$ is a direct sum of copies of $k$ and we may use $\psi$ to define a non-degenerate character of $\mathrm{U}_{n}(\mathbf{A})$ which is trivial on $\mathrm{U}_{n}(k)$. By abuse of notation we continue to call this character $\psi$.

The connected component of the Langlands dual group of $\mathrm{G}_{n}$ is ${ }^{\mathrm{L}} \mathrm{G}_{n}^{0}=\operatorname{Sp}_{2 n}(\mathbf{C})$. $\mathrm{GL}_{2 n}$ as an algebraic group over $k$ has as the connected component of its dual group ${ }^{\mathrm{L}} \mathrm{GL}_{2 n}^{0}=\mathrm{GL}_{2 n}(\mathbf{C})$. There is a natural embedding $\mathrm{\imath}:{ }^{\mathrm{L}} \mathrm{G}_{n}^{0}=\mathrm{Sp}_{2 n}(\mathbf{G}) \hookrightarrow{ }^{\mathrm{L}} \mathrm{GL}_{2 n}^{0}=\mathrm{GL}_{2 n}(\mathbf{G})$.

Let $\pi=\otimes^{\prime} \pi_{v}$ be an irreducible automorphic representation of $\mathrm{SO}_{2 n+1}(\mathbf{A})$.

For $v$ a finite place of $k$ where $\pi_{v}$ is unramified the representation $\pi_{v}$ of $\mathrm{G}_{n}\left(k_{v}\right)$ is completely determined by its Satake parameter, a semi-simple conjugacy class $\left[t_{v}\right]$ in ${ }^{\mathrm{L}} \mathrm{G}_{n}^{0}[29,2]$. $\left[t_{v}\right]$ then determines a semi-simple conjugacy class $\left[\mathrm{l}\left(t_{v}\right)\right]$ in ${ }^{\mathrm{L}} \mathrm{GL}_{2 n}^{0}$. An unramified irreducible admissible representation $\Pi_{v}$ of $\mathrm{GL}_{2 n}\left(k_{v}\right)$ is called the local Langlands lift of $\pi_{v}$ if its associated semi-simple conjugacy class in ${ }^{\mathrm{L}} \mathrm{GL}_{2 n}^{0}$ is $\left[\mathbf{l}\left(t_{v}\right)\right]$, or equivalently, $\mathrm{L}\left(s, \Pi_{v}\right)=\operatorname{det}\left(\mathrm{I}-t_{v} q_{v}^{-s}\right)^{-1}=\mathrm{L}\left(s, \pi_{v}\right)$.

If $v$ is an archimedean place, then by the arithmetic Langlands classification $\pi_{v}$ is determined by an admissible homomorphism $\varphi_{v}: \mathrm{W}_{v} \longrightarrow{ }^{\mathrm{L}} \mathrm{G}_{n}^{0}$ where $\mathrm{W}_{v}$ is the local Weil group of $k_{v}[23,2]$. The composition $\mathfrak{\imath} \circ \varphi_{v}$ is an admissible homomorphism of $\mathrm{W}_{v}$ into ${ }^{\mathrm{L}} \mathrm{GL}_{2 n}^{0}$ and hence determines a representation $\Pi_{v}$ of $\mathrm{GL}_{2 n}\left(k_{v}\right)$ such that $\mathrm{L}\left(s, \Pi_{v}\right)=\mathrm{L}\left(s, \pi_{v}\right)$. This is again the local lift of $\pi_{v}$.

An irreducible automorphic representation $\Pi=\otimes^{\prime} \Pi_{v}$ of $\mathrm{GL}_{2 n}(\mathbf{A})$ is called a weak Langlands lift of $\pi$, or for brevity simply a weak lift of $\pi$, if for every archimedean place $v$ and for almost all non-archimedean places $v$ for which $\pi_{v}$ is unramified we have that $\Pi_{v}$ is a local Langlands lift of $\pi_{v}$. In particular this entails an equality of (partial) Langlands L-functions $\mathrm{L}^{\mathrm{S}}(s, \Pi)=\prod_{v \notin \mathrm{S}} \mathrm{L}\left(s, \Pi_{v}\right)=\prod_{v \notin \mathrm{S}} \mathrm{L}\left(s, \pi_{v}\right)=\mathrm{L}^{\mathrm{S}}(s, \pi)$.

Let $\pi$ be an irreducible cuspidal representation of $\mathrm{G}_{n}(\mathbf{A})$. We say that $\pi$ is globally generic if there is a cusp form $\varphi \in \mathrm{V}_{\pi}$ such that $\varphi$ has a non-vanishing $\psi$-Fourier coefficient along $\mathrm{U}_{n}$, i.e., such that

$$
\int_{\mathrm{U}_{n}(k) \backslash \mathrm{U}_{n}(\mathbf{A})} \varphi(u g) \Psi^{-1}(u) d u \neq 0 .
$$

Cuspidal automorphic representations of $\mathrm{GL}_{n}$ are always globally generic in this sense. For cuspidal automorphic representations of $\mathrm{SO}_{2 n+1}$ this is a condition.

Theorem. - Let $k$ be a number field and let $\pi$ be an irreducible globally generic cuspidal automorphic representation of the split $\mathrm{SO}_{2 n+1}(\mathbf{A})$. Then $\pi$ has a weak lift to $\mathrm{GL}_{2 n}(\mathbf{A})$.

The proof of this theorem will be given in Section 5 after we develop some necessary preliminaries. 


\section{The Converse Theorem for $G L_{N}$}

In order to effect the weak lifting from $\mathrm{SO}_{2 n+1}$ to $\mathrm{GL}_{2 n}$ we will use the converse theorem for $\mathrm{GL}_{\mathrm{N}}$ with $\mathrm{N}=2 n$ in the following form. The applicability of the theorem in this form to the problem of lifting was outlined in [8].

Let us fix a finite set $\mathrm{S}$ of finite places of $k$.

For each integer $r$, let

$\mathscr{b}_{\mathrm{S}}(r)=\left\{\pi: \pi\right.$ is an irreducible generic automorphic representation of $\mathrm{GL}_{r}(\mathbf{A})$ such that the local component $\pi_{v}$ is unramified for all $\left.v \in \mathrm{S}\right\}$.

Similarly, let $\mathscr{C}_{\mathrm{S}}^{\circ}(r)$ be the cuspidal elements of $\mathscr{b}_{\mathrm{S}}(r)$. Let $\mathscr{T}(\mathrm{S})=\coprod_{r=1}^{\mathrm{N}-1} \cdot \mathscr{b}_{\mathrm{S}}^{\circ}(r)$. If $\eta$ is a continuous character of $k^{\times} \backslash \mathbf{A}^{\times}$, let $\mathscr{T}(\mathrm{S} ; \eta)=\mathscr{T}(\mathrm{S}) \otimes \eta=\left\{\tau=\tau^{\prime} \otimes \eta: \quad \tau^{\prime} \in \mathscr{T}(\mathrm{S})\right\}$.

Converse Theorem. - Let $\Pi=\otimes^{\prime} \Pi_{v}$ be an irreducible admissible representation of $\mathrm{GL}_{\mathrm{N}}(\mathbf{A})$ whose central character $\omega_{\Pi}$ is invariant under $k^{\times}$and whose $\mathrm{L}$-function $\mathrm{L}(s, \Pi)=\prod_{v} \mathrm{~L}\left(s, \Pi_{v}\right)$ is absolutely convergent in some right half plane. Let $\mathrm{S}$ be a finite set of finite places of $k$ and let $\eta$ be a continuous character of $k^{\times} \backslash \mathbf{A}^{\times}$. Suppose that for every $\tau \in \mathscr{T}(\mathrm{S} ; \eta)$ the $\mathrm{L}$-function $\mathrm{L}(s, \Pi \times \tau)$ satisfies

(1) $\mathrm{L}(s, \Pi \times \tau)$ and $\mathrm{L}(s, \widetilde{\Pi} \times \widetilde{\tau})$ extend to entire functions of $s \in \mathbf{G}$

(2) $\mathrm{L}(s, \Pi \times \tau)$ and $\mathrm{L}(s, \widetilde{\Pi} \times \widetilde{\tau})$ are bounded in vertical strips

(3) $\mathrm{L}(s, \Pi \times \tau)$ satisfies the functional equation $\mathrm{L}(s, \Pi \times \tau)=\varepsilon(s, \Pi \times \tau) \mathrm{L}(1-s, \widetilde{\Pi} \times \widetilde{\tau})$.

Then there exists an automorphic representation $\Pi^{\prime}$ of $\mathrm{GL}_{\mathrm{N}}(\mathbf{A})$ such that $\Pi_{v} \simeq \Pi_{v}^{\prime}$ for almost all v. More precisely, $\Pi_{v} \simeq \Pi_{v}^{\prime}$ for all $v \notin \mathrm{S}$.

In the statement of the theorem, the twisted L- and $\varepsilon$-factors are defined by the products

$$
\mathrm{L}(s, \Pi \times \tau)=\prod_{v} \mathrm{~L}\left(s, \Pi_{v} \times \tau_{v}\right) \quad \varepsilon(s, \Pi \times \tau)=\prod_{v} \varepsilon\left(s, \Pi_{v} \times \tau_{v}, \Psi_{v}\right)
$$

of local factors as in [4].

Proof. - This formulation of the converse theorem has not appeared in print. If the character $\eta$ is not present, i.e., it is stated in terms of the $\mathscr{T}(\mathrm{S})$ only, it is trivially a consequence of Theorem 2 of [7], which has the same statement but with twists only for $1 \leqslant r \leqslant \mathrm{~N}-2$ (assuming $\mathrm{N} \geqslant 3$ ). The proof of our current statement is simpler than that of Theorem 2 of [7] and can be given along the lines of Theorem 1 of [4]. We will sketch the necessary modifications to the proof of Theorem 1 of [4] for the convenience of the reader. We will follow the notational conventions [4] without comment. For the duration of this proof we let $\mathrm{G}=\mathrm{GL}_{\mathrm{N}}$. We follow the standard 
convention that for a set of places $\mathrm{S}$ we let $\mathrm{G}_{\mathrm{S}}=\prod_{v \in \mathrm{S}} \mathrm{G}_{v}$ and $\mathrm{G}^{\mathrm{S}}=\prod_{v \notin \mathrm{S}}^{\prime} \mathrm{G}_{v}$ with similar conventions for representations, L-functions, etc.

We begin with $\Pi=\otimes^{\prime} \Pi_{v}$ as in the theorem. We assume that $\mathrm{L}(s, \Pi \times \tau)$ is nice, that is, satisfies (1)-(3) of the theorem, for all $\tau \in \mathscr{T}(\mathrm{S})$. For each place $v$, if $\Pi_{v}$ is not generic we take an induced representation $\Xi_{v}$ of Whittaker type such that $\Pi_{v}$ is the unique Langlands quotient of $\Xi_{v}$. For those places where $\Pi_{v}$ is generic, $\Xi_{v}=\Pi_{v}$. For those places where $\Pi_{v}$ is unramified, we choose an unramified vector $\xi_{v}^{\circ}$ which projects to the unique non-zero $\mathbf{K}_{v}$-fixed vector in $\Pi_{v}$ with respect to which the restricted tensor product is taken. We then form $\Xi=\otimes^{\prime} \Xi_{v}$, the restricted tensor product with respect to this system $\left\{\xi_{v}^{\circ}\right\}$. By the definition of the local L- and $\varepsilon$-factors [13, 17] we have both $\mathrm{L}(s, \Xi \times \tau)=\mathrm{L}(s, \Pi \times \tau)$ and $\varepsilon(s, \Xi \times \tau)=\varepsilon(s, \Pi \times \tau)$ for all $\tau$.

Since representations of Whittaker type have Whittaker models, for each decomposable $\xi \in \mathrm{V}_{\Xi}, \xi=\otimes \xi_{v}$, we may form $\mathrm{W}_{\xi}(g)=\prod \mathrm{W}_{\xi_{v}}\left(g_{v}\right)$ and the functions

$$
\mathrm{U}_{\xi}(g)=\sum_{\mathrm{N}(k) \backslash \mathrm{P}(k)} \mathrm{W}_{\xi}(p g) \quad \text { and } \quad \mathrm{V}_{\xi}(g)=\sum_{\mathrm{N}^{\prime}(k) \backslash \mathrm{Q}(k)} \mathrm{W}_{\xi}(\alpha q g)
$$

where $\mathrm{P}=\mathrm{P}_{\mathrm{N}}$ is the standard mirabolic subgroup fixing the row vector $(0, \ldots, 0,1), \mathrm{N}$ is the standard upper triangular maximal unipotent subgroup of $\mathrm{GL}_{\mathrm{N}}, \mathrm{Q}$ is the transpose of $\mathrm{P}$, that is, the opposite mirabolic, $\alpha$ is the permutation matrix $\alpha=\left(\mathrm{I}_{\mathrm{N}-1} \quad 1\right)$, and $\mathrm{N}^{\prime}=\alpha^{-1} \mathrm{~N} \alpha$. Since $\mathrm{P}(k)$ and $\mathrm{Q}(k)$ generate $\mathrm{GL}_{\mathrm{N}}(k)$ we see that an equality $\mathrm{U}_{\xi}(g)=\mathrm{V}_{\xi}(g)$ for all $\xi$ would imply that the map $\xi \mapsto \mathrm{U}_{\xi}(g)$ would embed $\mathrm{V}_{\Xi}$ into the space of automorphic forms on $\mathrm{GL}_{\mathrm{N}}(\mathbf{A})$.

Since we are only twisting by representations which are unramified at places in $\mathrm{S}$, we will only be able to prove this equality for a restricted set of $\xi$ and only on a subset of $\mathrm{GL}_{\mathrm{N}}(\mathbf{A})$. For every place $v \in \mathrm{S}$ we choose a vector $\xi_{v}^{\circ}$ such that $\xi_{v}^{\circ}$ is invariant under some compact open subgroup $\mathrm{K}_{1}\left(\mathfrak{p}_{v}^{m_{v}}\right)$ with $m_{v} \geqslant 0$ (see Section 11 of [4]). This vector will necessarily transform by the character $\omega_{\Pi_{v}}$ under the action of $\mathbf{K}_{0}\left(\mathfrak{p}_{v}^{m_{v}}\right)$. If $v$ happens to be an unramified place, we take $\xi_{v}^{\circ}$ to be the $\mathbf{K}_{v}$-fixed vector as before. Let $\xi_{\mathrm{S}}^{\circ}=\otimes_{v \in \mathrm{S}} \xi_{v}^{\circ} \in \mathrm{V}_{\Xi_{\mathrm{S}}}$. Let $\mathrm{K}_{0, \mathrm{~S}}(\mathfrak{n})=\prod_{v \in \mathrm{S}} \mathrm{K}_{0, v}\left(\mathfrak{p}_{v}^{m_{v}}\right) \subset \mathrm{G}_{\mathrm{S}}$ where $\mathfrak{n}=\prod_{v \in \mathrm{S}} \mathfrak{p}_{v}^{m_{v}}$.

Let $\xi^{\mathrm{S}}$ be any vector in $\mathrm{V}_{\Xi} \mathrm{s}$. Then as in Section 10 of [4] for $\xi$ of the form $\xi=\xi_{\mathrm{S}}^{\circ} \otimes \xi^{\mathrm{S}}$ the fact that the L-functions for $\mathrm{L}(s, \Xi \times \tau)$ are nice for all $\tau \in \mathscr{T}(\mathrm{S})$ lets us conclude that $\mathrm{U}_{\xi}(g)=\mathrm{V}_{\xi}(g)$ for all $g \in \mathrm{K}_{0, \mathrm{~S}}(\mathfrak{n}) \mathrm{G}^{\mathrm{S}}$.

Let $\mathrm{P}_{0}(\mathfrak{n})=\mathrm{P}(k) \cap \mathrm{K}_{0, \mathrm{~s}}(\mathfrak{n}) \mathrm{G}^{\mathrm{S}}$ and $\mathrm{Q}_{0}(\mathfrak{n})=\mathrm{Q}(k) \cap \mathrm{K}_{0, \mathrm{~s}}(\mathfrak{n}) \mathrm{G}^{\mathrm{S}}$. Then Proposition 9.1 of [4] is replaced by a simple matrix computation (as in Lemma 2 of Section 4 of [7]) which shows that $\mathrm{P}_{0}(\mathfrak{n})$ and $\mathrm{Q}_{0}(\mathfrak{n})$ generate the congruence type subgroup $\Gamma_{0}(\mathfrak{n})=\mathrm{GL}_{\mathrm{N}}(k) \cap \mathrm{K}_{0, \mathrm{~S}}(\mathfrak{n}) \mathrm{G}^{\mathrm{S}}$ of $\mathrm{G}^{\prime}=\mathrm{K}_{0, \mathrm{~S}}(\mathfrak{n}) \mathrm{G}^{\mathrm{S}}$. Hence the mapping $\xi^{\mathrm{S}} \mapsto \mathrm{U}_{\xi_{\mathrm{S}} \otimes \xi^{\mathrm{S}}}(g)$ embeds $V_{\Xi}$ s into the space of automorphic forms $\mathscr{C}\left(\Gamma_{0}(\mathfrak{n}) \backslash G^{\prime} ; \omega_{\Xi}\right)$ as a representation of $\mathrm{G}^{\prime}$. Since by approximation $\mathrm{GL}_{\mathrm{N}}(\mathbf{A})=\mathrm{GL}_{\mathrm{N}}(k) \mathrm{G}^{\prime}$ and $\Gamma_{0}(\mathfrak{n})=\mathrm{GL}_{\mathrm{N}}(k) \cap \mathrm{G}^{\prime}$ we see 
that $\mathscr{C}\left(\mathrm{GL}_{\mathrm{N}}(k) \backslash \mathrm{GL}_{\mathrm{N}}(\mathbf{A}) ; \omega_{\Xi}\right)=\mathscr{\mathscr { C }}\left(\Gamma_{0}(\mathfrak{n}) \backslash \mathrm{G}^{\prime} ; \omega_{\Xi}\right)$ so that $\Xi^{\mathrm{S}}$ determines an automorphic representation $\Xi^{\prime}$ of $\mathrm{GL}_{\mathrm{N}}(\mathbf{A})$. Then, by construction, $\Xi_{v}^{\prime} \simeq \Xi_{v}$ for all $v \notin \mathrm{S}$.

For our $\Pi^{\prime}$ of the theorem we now take any irreducible constituent of $\Xi^{\prime}$ but with the restriction that for $v \notin \mathrm{S}$ we take $\Pi_{v}^{\prime}=\Pi_{v}$, which is possible by our construction. This proves the result without the character $\eta$.

To incorporate the character $\eta$, the only thing to observe is that if $\tau \in \mathscr{T}(\mathrm{S})$ then $\mathrm{L}(s, \Pi \times(\tau \otimes \eta))=\mathrm{L}(s,(\Pi \otimes \eta) \times \tau)$ so that applying the converse theorem for $\Pi$ with twisting sets $\mathscr{T}(\mathrm{S} ; \eta)$ is equivalent to applying the converse theorem for $\Pi \otimes \eta$ with the twisting sets $\mathscr{T}(\mathrm{S})$. Then by the above, $\Pi \otimes \eta$ is quasi-automorphic and hence $\Pi$ is as well.

To apply this theorem to the problem of Langlands lifting form $\mathrm{SO}_{2 n+1}$ to $\mathrm{GL}_{2 n}$, we begin with our globally generic cuspidal automorphic representation $\pi=\otimes^{\prime} \pi_{v}$ of $\mathrm{SO}_{2 n+1}(\mathbf{A})$. For each place $v$ we need to associate to $\pi_{v}$ an irreducible admissible representation $\Pi_{v}$ of $\mathrm{GL}_{2 n}\left(k_{v}\right)$ such that for every $\tau \in \mathscr{T}(\mathrm{S} ; \eta)$ we have

$$
\begin{aligned}
\mathrm{L}\left(s, \pi_{v} \times \tau_{v}\right) & =\mathrm{L}\left(s, \Pi_{v} \times \tau_{v}\right) \\
\varepsilon\left(s, \pi_{v} \times \tau_{v}, \psi_{v}\right) & =\varepsilon\left(s, \Pi_{v} \times \tau_{v}, \psi_{v}\right) .
\end{aligned}
$$

For archimedean places $v$ and those non-archimedean $v$ where $\pi_{v}$ is unramified, we take $\Pi_{v}$ to be the local Langlands lift of $\pi_{v}$. For those places $v$ where $\pi_{v}$ is ramified, we will take for $\Pi_{v}$ an essentially arbitrary irreducible admissible generic representation of $\mathrm{GL}_{2 n}\left(k_{v}\right)$ having trivial central character. However, we must choose our finite set of places $\mathrm{S}$ of $k$ such that $\mathrm{S}$ contains the places where $\pi_{v}$ is ramified and choose our character $\eta$ of $k^{\times} \backslash \mathbf{A}^{\times}$such that $\eta_{v}$ is sufficiently highly ramified so that $\mathrm{L}\left(s, \pi_{v} \times \eta_{v}\right)$, $\mathrm{L}\left(s, \Pi_{v} \times \eta_{v}\right), \quad \varepsilon\left(s, \pi_{v} \times \eta_{v}, \boldsymbol{\psi}_{v}\right)$, and $\varepsilon\left(s, \Pi_{v} \times \eta_{v}, \psi_{v}\right)$ are all standard (see Section 4).

Now consider $\Pi=\otimes^{\prime} \Pi_{v}$. This is an irreducible representation of $\mathrm{GL}_{2 n}(\mathbf{A})$. With the choices above we have

$$
\begin{aligned}
\mathrm{L}(s, \pi \times \tau) & =\mathrm{L}(s, \Pi \times \tau) \\
\varepsilon(s, \pi \times \tau) & =\varepsilon(s, \Pi \times \tau)
\end{aligned}
$$

for $\operatorname{Re}(s)>>0$ and all $\tau \in \mathscr{T}(\mathrm{S} ; \eta)$. In the next section we will show that the theory of L-functions for $\mathrm{SO}_{2 n+1} \times \mathrm{GL}_{r}$ is developed far enough to guarantee that $\Pi$ satisfies the hypotheses of this converse theorem. Hence $\Pi$ is quasi-automorphic and moreover there exists an irreducible automorphic representation $\Pi^{\prime}$ of $\mathrm{GL}_{2 n}(\mathbf{A})$ such that $\Pi_{v} \simeq \Pi_{v}^{\prime}$ for all archimedean $v$ and almost all finite $v$ where $\pi_{v}$ is unramified. Hence $\Pi^{\prime}$ is a weak Langlands lift of $\pi$. 


\section{Analytic properties of L-functions $\mathrm{SO}_{2 n+1}$ twisted by $\mathrm{GL}_{r}$}

Let $\pi$ be a globally generic cuspidal representation of $\mathrm{G}_{n}(\mathbf{A})=\mathrm{SO}_{2 n+1}(\mathbf{A})$.

For $\tau$ a cuspidal representation of $\operatorname{GL}_{r}(\mathbf{A})$ we will let $\mathrm{L}(s, \pi \times \tau)$ be the completed L-function as defined in [31] via the theory of Eisenstein series. The local factors are then defined via the arithmetic Langlands classification for archimedean places, through the Satake parameters for finite unramified places, as the poles of the associated $\gamma$-factor (or local coefficient) if $\pi_{v}$ and $\tau_{v}$ are tempered, by analytic extension if $\pi_{v}$ and $\tau_{v}$ are quasi-tempered, and via the representation theoretic Langlands classification otherwise.

Let $\mathrm{S}$ be a non-empty set of finite places of $k$ such that $\pi_{v}$ is unramified for all finite $v \notin$ S. Let $\eta$ be a continuous character of $k^{\times} \backslash \mathbf{A}^{\times}$which is highly ramified for all $v \in \mathrm{S}$.

Proposition 3.1. - Let $\eta$ be a character of $k^{\times} \backslash \mathbf{A}^{\times}$such that, for some $v \in \mathrm{S}$, both $\eta_{v}$ and $\eta_{v}^{2}$ are ramified. Then for all $\tau \in \mathscr{T}(\mathrm{S} ; \eta)$ the $\mathrm{L}$-function $\mathrm{L}(s, \pi \times \tau)$ is entire.

This follows from the results in $[18,19]$. For the convenience of the reader, we will sketch the proof here.

Let $\mathbf{A}^{\times, 1}$ denote the group of ideles of norm 1. Fix a subgroup $A_{+} \subset \mathbf{A}^{\times}$such that $\mathrm{A}_{+} \simeq \mathbf{R}_{+}^{\times}$and $\mathbf{A}^{\times}=\mathbf{A}^{\times, 1} \times \mathrm{A}_{+}$. It suffices to assume that $\tau$ is unitary and its central character is a character of $k^{\times} \backslash \mathbf{A}^{\times}$which is trivial on $\mathrm{A}_{+}$. Any cuspidal representation can be written as $\left.\tau \simeq \tau^{\prime} \otimes|\operatorname{det}|\right|^{\prime}$, where $\tau^{\prime}$ is unitary with central character trivial on $\mathrm{A}_{+}$, and then $\mathrm{L}(s, \pi \times \tau)=\mathrm{L}\left(s+s^{\prime}, \pi \times \tau^{\prime}\right)$. Note that if $\tau \in \mathscr{T}(\mathrm{S} ; \eta)$, then so is $\tau^{\prime}$.

Let $\pi$ be as above and $\tau$ a cuspidal representation of $\mathrm{GL}_{r}(\mathbf{A})$ in $\mathscr{T}(\mathrm{S} ; \boldsymbol{\eta})$ also as above. If we let $\mathrm{M}=\mathrm{GL}_{r} \times \mathrm{G}_{n}$ then $\mathrm{M}$ is a Levi subgroup of a standard maximal parabolic subgroup $\mathrm{P}=\mathrm{P}_{r, n} \subset \mathrm{G}_{r+n}$. Let $m=r+n$ and let $\mathrm{N}=\mathrm{N}_{r, n}$ be the unipotent radical of $\mathrm{P}$. In order to make the argument, we will consider both the case $n \neq 0$, the case of interest, and $n=0$, for purposes of induction. We view $\sigma=\widetilde{\tau} \otimes \pi$ as a unitary cuspidal globally generic representation of $\mathbf{M}(\mathbf{A})$. As such, we can form the induced representation

$$
\mathrm{I}(s, \sigma)= \begin{cases}\operatorname{Ind}_{\mathrm{P}(\mathbf{A})}^{\mathrm{G}_{m}(\mathbf{A})}\left(|\operatorname{det}|^{s} \widetilde{\tau} \otimes \pi\right) & \text { if } \quad n \neq 0 \\ \operatorname{Ind}_{\mathrm{P}(\mathbf{A})}^{\mathrm{G}_{m}(\mathbf{A})}\left(|\operatorname{det}|^{s / 2} \widetilde{\tau}\right) & \text { if } \quad n=0\end{cases}
$$

If $\alpha$ is the simple root associated to the maximal parabolic subgroup $\mathrm{P}$ and we let, as usual, $\widetilde{\alpha}=\rho_{\mathrm{P}} /\left\langle\rho_{\mathrm{P}}, \alpha\right\rangle$ then as in [31]

$$
\mathrm{I}(s, \boldsymbol{\sigma})=\operatorname{Ind}_{\mathrm{P}(\mathbf{A})}^{\mathrm{G}_{m}(\mathbf{A})}\left(e^{\left\langle s \widetilde{\alpha}, \mathrm{H}_{\mathrm{P}}\right\rangle} \boldsymbol{\sigma}\right) .
$$

We let $\mathrm{V}(s, \boldsymbol{\sigma})$ denote the space of functions on which $\mathrm{I}(s, \boldsymbol{\sigma})$ acts. We could represent these representations as all realized on the same underlying space of functions on a 
suitable maximal compact subgroup $\mathrm{K}(\mathbf{A})$, namely the space $\mathrm{V}$ of

$$
\operatorname{Ind}_{\mathrm{K}(\mathbf{A}) \cap \mathrm{P}(\mathbf{A})}^{\mathrm{K}(\mathbf{A})}\left(\left.\boldsymbol{\sigma}\right|_{\mathrm{K}(\mathbf{A}) \cap \mathrm{M}(\mathbf{A})}\right)
$$

with the action varying with $s$. Each $f \in \mathrm{V}$ then determines a section $f_{s} \in \mathrm{V}(s, \sigma)$.

Consider, for $f \in \mathrm{V}$, the Eisenstein series

$$
\mathrm{E}\left(g, f_{s}\right)=\sum_{\mathrm{P}(k) \backslash \mathrm{G}_{m}(k)} f_{s}(\gamma g) .
$$

This converges for $\operatorname{Re}(s)>>0$ and has a meromorphic continuation to all of $\mathbf{C}$ [21, 26]. Our condition on the central character of $\tau$ guarantees that the poles of the Eisenstein series in the half plane $\operatorname{Re}(s) \geqslant 0$ are all simple and lie on the real axis.

The first key observation is the following.

Lemma 3.1. - If $\tau$ is not self-contragredient then $\mathrm{E}\left(g, f_{s}\right)$ has no poles in $\operatorname{Re}(s) \geqslant 0$.

Proof. - This is Proposition 2.1 of [18]. It follows from Langlands' inner product formula for the residues of Eisenstein series [21, 26].

This will hold in our situation by the following lemma.

Lemma 3.2. - Let $\tau \in \mathscr{T}(\mathrm{S} ; \eta)$ with $\eta$ non-trivial and such that for some $v \in \mathrm{S}, \eta_{v}^{2}$ is ramified. Then $\tau$ cannot be self-contragredient.

Proof. - If $\tau \in \mathscr{T}(\mathrm{S} ; \eta)$ then at a place $v \in \mathrm{S}$ we have that the local component $\tau_{v}$ must be of the form

$$
\tau_{v} \simeq \operatorname{Ind}_{\mathrm{B}_{r}^{\prime}\left(k_{v}\right)}^{\mathrm{GL}_{r}\left(k_{v}\right)}\left(\mu_{1, v} \otimes \ldots \otimes \mu_{r, v}\right) \otimes \eta_{v} \simeq \operatorname{Ind}_{\mathrm{B}_{r}^{\prime}\left(k_{v}\right)}^{\mathrm{GL}_{v}\left(k_{v}\right)}\left(\mu_{1, v} \eta_{v} \otimes \ldots \otimes \mu_{r, v} \eta_{v}\right)
$$

where $\mathrm{B}_{r}^{\prime}$ is the upper triangular Borel subgroup of $\mathrm{GL}_{r}$ and the $\mu_{i, v}$ are unramified characters. This follows from the fact that $\tau_{v}=\tau_{v}^{\prime} \otimes \eta_{v}$ with $\tau_{v}^{\prime}$ both unramified and generic. Then

$$
\widetilde{\tau}_{v} \simeq \operatorname{Ind}_{B_{r}^{\prime}\left(k_{v}\right)}^{\mathrm{GL}_{v}\left(k_{v}\right)}\left(\mu_{1, v}^{-1} \eta_{v}^{-1} \otimes \ldots \otimes \mu_{r, v}^{-1} \eta_{v}^{-1}\right)
$$

If $\widetilde{\tau} \simeq \tau$ then for this place $\widetilde{\tau}_{v} \simeq \tau_{v}$ and then for each $i$ there will be a $j$ such that $\mu_{i, v} \eta_{v}=\mu_{j, v}^{-1} \eta_{v}^{-1}$ and hence $\eta_{v}^{2}=\left(\mu_{j, v} \mu_{i, v}\right)^{-1}$. But $\eta_{v}^{2}$ is non-trivial and ramified while $\left(\boldsymbol{\mu}_{j, v} \boldsymbol{\mu}_{i, v}\right)^{-1}$ is unramified. This is a contradiction.

From this point on we will use simply that $\tau$ is not self-contragredient.

Let $\Sigma$ denote the set of roots of $\mathrm{G}_{m}, \Sigma^{+}$the set of positive roots associated to $\mathrm{U}_{m}$, and $\Delta$ denote the associated set of simple roots. Let $\theta$ be the subset of $\Delta$ which 
generates $M$. Then $\Delta=\theta \cup\{\alpha\}$. Let $w$ be the unique element of the Weyl group satisfying $w(\boldsymbol{\theta}) \subset \Delta$ but $w(\boldsymbol{\alpha}) \in \Sigma^{-}$, i.e., $w(\boldsymbol{\alpha})$ is negative. In our setting we have

$$
w=\left(\begin{array}{ccc} 
& & s_{r} \\
& \pm \mathrm{I}_{2 n+1} & \\
s_{r} & &
\end{array}\right)
$$

where $s_{r}$ is the $r \times r$ matrix

$$
s_{r}=\left(\begin{array}{lll} 
& & 1 \\
& . & \\
1 & &
\end{array}\right)
$$

and the sign on $\pm \mathrm{I}_{2 n+1}$ is chosen so that the determinant is 1 . Note that $w$ preserves $\mathrm{M}$ and that if we define $w \boldsymbol{\sigma}(m)=\boldsymbol{\sigma}\left(m^{w}\right)$ then $w \boldsymbol{\sigma} \simeq \tau \otimes \pi$ and $w\left(e^{\left\langle s \widetilde{\alpha}, \mathrm{H}_{\mathrm{P}}\right\rangle} \boldsymbol{\sigma}\right)=e^{\left\langle-s \widetilde{\alpha}, \mathrm{H}_{\mathrm{P}}\right\rangle} w \boldsymbol{\sigma}$. We let $\mathrm{A}(s, \sigma, w): \mathrm{V}(s, \sigma) \longrightarrow \mathrm{V}(-s, w \sigma)$ be the standard intertwining operator defined for $\operatorname{Re}(s)>>0$ by

$$
\mathrm{A}(s, \sigma, w) f_{s}(g)=\int_{\mathrm{N}(\mathbf{A})} f_{s}(w n g) d n
$$

and continued meromorphically (see [31] and the references therein). $\mathrm{A}(s, \sigma, w)$ factors into a product of local intertwining operators $\mathrm{A}(s, \sigma, w)=\otimes_{v} \mathrm{~A}\left(s, \sigma_{v}, w\right)$ with the local operators given by the analogous local integrals. These local operators can be normalized by writing

$$
\mathrm{A}\left(s, \sigma_{v}, w\right)=r\left(s, \sigma_{v}, w\right) \mathrm{N}\left(s, \sigma_{v}, w\right)
$$

where, if $n \neq 0$,

$$
r\left(s, \sigma_{v}, w\right)=\frac{\mathrm{L}\left(s, \pi_{v} \times \tau_{v}\right) \mathrm{L}\left(2 s, \tau_{v}, \operatorname{Sym}^{2}\right)}{\mathrm{L}\left(1+s, \pi_{v} \times \tau_{v}\right) \varepsilon\left(s, \pi_{v} \times \tau_{v}, \psi_{v}\right) \mathrm{L}\left(1+2 s, \tau_{v}, \operatorname{Sym}^{2}\right) \varepsilon\left(2 s, \tau_{v}, S_{y y m}^{2}, \psi_{v}\right)}
$$

while if $n=0$,

$$
r\left(s, \sigma_{v}, w\right)=r\left(s, \tau_{v}, w\right)=\frac{\mathrm{L}\left(s, \tau_{v}, \operatorname{Sym}^{2}\right)}{\mathrm{L}\left(1+s, \tau_{v}, \operatorname{Sym}^{2}\right) \varepsilon\left(s, \tau_{v}, \operatorname{Sym}^{2}, \boldsymbol{\psi}_{v}\right)}
$$

are scalar functions. The $\mathrm{N}\left(s, \sigma_{v}, w\right)$ are then the normalized intertwining operators. Note that if $v$ is a non-archimedean place where $\mathrm{I}\left(s, \sigma_{v}\right)$ is unramified and we let $f_{v, s}^{\circ}$ denote the (normalized) $\mathbf{K}_{v}$-fixed vector in $\mathrm{V}\left(s, \sigma_{v}\right)$ and similarly $\tilde{f}_{v,-s}^{\circ}$ the $\mathbf{K}_{v}$ fixed vector of $\mathrm{V}\left(-s, w \sigma_{v}\right)$ then $\mathrm{N}\left(s, \sigma_{v}, w\right) f_{v, s}^{\circ}=\widetilde{f}_{v,-s}^{\circ}$ by the formula of Gindikin and Karpelevich. Hence if we set $\mathrm{N}(s, \sigma, w)=\otimes_{v} \mathrm{~N}\left(s, \sigma_{v}, w\right)$ then for any $f_{s} \in \mathrm{V}(s, \sigma)$, $\mathrm{N}(s, \sigma, w) f_{s}$ is essentially a finite product. 
Lemma 3.3. — Each normalized local intertwining operator $\mathrm{N}\left(s, \sigma_{v}, w\right)$ is holomorphic and non-zero (as an operator) for $\operatorname{Re}(s) \geqslant \frac{1}{2}$. Consequently the same is true for the global operator $\mathrm{N}(s, \sigma, w)$.

Proof. - This is Proposition 3.4 of [19].

Now let $r(s, \sigma, w)=\prod_{v} r\left(s, \sigma_{v}, w\right)$. The Euler products converges for $\operatorname{Re}(s)>>0$. If we compute the constant term of the Eisenstein series along the unipotent radical $\mathrm{N}$ of $\mathrm{P}$ we have

$$
\mathrm{E}^{\mathrm{N}}\left(g, f_{s}\right)=\int_{\mathrm{N}(k) \backslash \mathrm{N}(\mathbf{A})} \mathrm{E}\left(n g, f_{s}\right) d n=f_{s}(g)+\mathrm{A}(s, \sigma, w) f_{s}(g) .
$$

By Lemma 3.1 and 3.2, the Eisenstein series is holomorphic for $\operatorname{Re}(s) \geqslant 0$, and hence the same is true of $\mathrm{A}(s, \sigma, w)$. So if we write

$$
\mathrm{A}(s, \sigma, w)=r(s, \sigma, w) \mathrm{N}(s, \sigma, w)
$$

then by Lemma 3.3 we may conclude that $r(s, \sigma, w)$ is holomorphic for $\operatorname{Re}(s) \geqslant \frac{1}{2}$, of course for $\tau$ non-self-contragredient.

From this we need to sift out our information on $\mathrm{L}(s, \pi \times \tau)$. Let us write $r(s, \sigma, w)=p(s, \sigma, w) / q(s, \sigma, w)$ where

$$
p(s, \sigma, w)= \begin{cases}\mathrm{L}(s, \pi \times \tau) \mathrm{L}\left(2 s, \tau, S_{y m^{2}}\right) & \text { if } n \neq 0 \\ \mathrm{~L}\left(s, \tau, \operatorname{Sym}^{2}\right) & \text { if } n=0\end{cases}
$$

and

$$
q(s, \sigma, w)= \begin{cases}\mathrm{L}(1+s, \pi \times \tau) \varepsilon(s, \pi \times \tau) \mathrm{L}\left(1+2 s, \tau, \operatorname{Sym}^{2}\right) \varepsilon\left(2 s, \tau, \operatorname{Sym}^{2}\right) & \text { if } n \neq 0 \\ \mathrm{~L}\left(s, \tau, \operatorname{Sym}^{2}\right) \varepsilon\left(s, \tau, \operatorname{Sym}^{2}\right) & \text { if } n=0\end{cases}
$$

with similar conventions in the local situation.

If we compute a non-trivial Fourier coefficient of our Eisenstein series we find, as in Proposition 5.2 of [10],

$$
\mathrm{E}^{\psi}\left(e, f_{s}\right)=\int_{\mathrm{U}_{m}(k) \backslash \mathrm{U}_{m}(\mathbf{A})} \mathrm{E}\left(u, f_{s}\right) \psi^{-1}(u) d u=q^{\mathrm{T}}(s, \sigma, w)^{-1} \prod_{v \in \mathrm{T}} \mathrm{W}_{v, s}(e)
$$

where $\mathrm{U}_{m}$ is the standard maximal unipotent subgroup of $\mathrm{G}_{m}, \psi$ our non-trivial additive character of $k \backslash \mathbf{A}$ defining a non-degenerate character of $\mathbf{U}_{m}(k) \backslash \mathbf{U}_{m}(\mathbf{A})$ which we again denote by $\psi, \mathrm{T}$ the finite set of places, $\mathrm{T} \supset \mathrm{S} \cup \mathrm{S}_{\infty}$, such that $\sigma$ and $\psi$ are both unramified for $v \notin \mathrm{T}, q^{\mathrm{T}}(s, \sigma, w)=\prod_{v \notin \mathrm{T}} q\left(s, \boldsymbol{\sigma}_{v}, w\right)$, and $\mathrm{W}_{v},{ }_{s}(g)$ simply local Whittaker functions for $\mathrm{I}\left(s, \sigma_{v}\right)$. Recall that for any given $s$ there is a choice of function so that 
$\mathrm{W}_{v, s}(e) \neq 0$, as in Lemma 5.3 of [10]. As a consequence of this formula and the fact that $\mathrm{E}\left(g, f_{s}\right)$ is holomorphic for $\operatorname{Re}(s) \geqslant 0$ we see that $q^{\mathrm{T}}(s, \sigma, w)^{-1}$ is holomorphic for $\operatorname{Re}(s) \geqslant 0$ and hence $q^{\mathrm{T}}(s, \sigma, w)$ is meromorphic and non-vanishing for $\operatorname{Re}(s) \geqslant 0$. Since the local L-factors are always meromorphic and non-vanishing, we have that in fact $q(s, \sigma, w)$ is meromorphic and non-vanishing for $\operatorname{Re}(s) \geqslant 0$. In particular, if $n=0$ this gives the meromorphy and non-vanishing of $\mathrm{L}\left(1+s, \tau, \operatorname{Sym}^{2}\right)$ for $\operatorname{Re}(s) \geqslant 0$, or of $\mathrm{L}\left(s, \tau, \operatorname{Sym}^{2}\right)$ for $\operatorname{Re}(s) \geqslant 1$.

Consider now the case $n=0$ so that $\sigma=\widetilde{\tau}$. We know that

$$
\mathrm{A}(s, \sigma, w)=r(s, \sigma, w) \mathrm{N}(s, \sigma, w)
$$

$\mathrm{A}(s, \sigma, w)$ is holomorphic for $\operatorname{Re}(s) \geqslant 0$ and $\mathrm{N}(s, \sigma, w)$ is holomorphic and nonvanishing for $\operatorname{Re}(s) \geqslant \frac{1}{2}$. Hence $r(s, \sigma, w)$ is holomorphic for $\operatorname{Re}(s) \geqslant \frac{1}{2}$. By the definition of $r(s, \sigma, w)$ we have

$$
\mathrm{L}\left(s, \tau, \operatorname{Sym}^{2}\right)=r(s, \sigma, w) \mathrm{L}\left(1+s, \tau, \operatorname{Sym}^{2}\right) \varepsilon\left(s, \tau, \operatorname{Sym}^{2}\right) .
$$

We know that the $\mathrm{L}\left(s, \tau, S y m^{2}\right)$ is absolutely convergent, and hence holomorphic, in some half plane, say $\operatorname{Re}(s)>a$. On the other hand, if $\mathrm{L}\left(s, \tau, \operatorname{Sym}^{2}\right)$ is holomorphic in $\operatorname{Re}(s)>b$ for some $b$, then $\mathrm{L}\left(1+s, \tau, \operatorname{Sym}^{2}\right)$ is holomorphic for $\operatorname{Re}(s)>b-1$ and hence so is $r(s, \tau, w) \mathrm{L}\left(1+s, \tau, \operatorname{Sym}^{2}\right) \varepsilon\left(s, \tau, \operatorname{Sym}^{2}\right)$ as long as $\operatorname{Re}(s) \geqslant \frac{1}{2}$. So $\mathrm{L}\left(s, \tau, \operatorname{Sym}^{2}\right)$ is then also holomorphic for $\operatorname{Re}(s)>b-1$ as long as $\operatorname{Re}(s) \geqslant \frac{1}{2}$. In this way we may inductively shift the half plane of holomorphy for $\mathrm{L}\left(s, \tau, S y m^{2}\right)$ to $\operatorname{Re}(s) \geqslant \frac{1}{2}$.

Now let $n \neq 0$. We proceed in essentially the same manner. From $\mathrm{A}(s, \sigma, w)$ $=r(s, \sigma, w) \mathrm{N}(s, \sigma, w)$ we may conclude that $r(s, \sigma, w)$ is holomorphic for $\operatorname{Re}(s) \geqslant \frac{1}{2}$. Utilizing the definition of $r(s, \sigma, w)$ we may write

$$
\mathrm{L}(s, \pi \times \tau)=\mathrm{R}(s) \mathrm{L}(1+s, \pi \times \tau)
$$

where we have written

$$
\mathrm{R}(s)=r(s, \sigma, w) \boldsymbol{\varepsilon}(s, \pi \times \tau) \mathrm{L}\left(1+2 s, \tau, \operatorname{Sym}^{2}\right) \boldsymbol{\varepsilon}\left(2 s, \tau, \operatorname{Sym}^{2}\right) \mathrm{L}\left(2 s, \tau, S y m^{2}\right)^{-1} .
$$

Note that from our previous analysis of the $n=0$ case we know that $\mathrm{L}\left(1+2 s, \tau, \operatorname{Sym}^{2}\right)$ is holomorphic for $\operatorname{Re}(s) \geqslant 0$ and $\mathrm{L}\left(2 s, \tau, S y m^{2}\right)^{-1}$ is holomorphic for $\operatorname{Re}(s) \geqslant \frac{1}{2}$. Hence $\mathrm{R}(s)$ is holomorphic for $\operatorname{Re}(s) \geqslant \frac{1}{2}$. Hence we can shift as above to obtain $\mathrm{L}(s, \pi \times \tau)$ 
is holomorphic for $\operatorname{Re}(s) \geqslant \frac{1}{2}$. We now utilize the functional equation (see Proposition 3.3 below) to guarantee $\mathrm{L}(s, \pi \times \tau)$ is entire. This proves Proposition 3.1.

We will also need the following two results on the analytic properties of these L-functions.

Proposition 3.2. - For any cuspidal representation $\tau$ of $\mathrm{GL}_{r}(\mathbf{A}), 1 \leqslant r<2 n$, the $\mathrm{L}-$ function $\mathrm{L}(s, \pi \times \tau)$ is bounded in vertical strips.

Proof. — This is Corollary 4.5 of [10].

Proposition 3.3. - For any cuspidal representation $\tau$ of $\mathrm{GL}_{r}(\mathbf{A}), 1 \leqslant r<2 n$, we have the functional equation

$$
\mathrm{L}(s, \pi \times \tau)=\varepsilon(s, \pi \times \tau) \mathrm{L}(1-s, \tilde{\pi} \times \tilde{\tau})
$$

Proof. - This is a consequence of Theorem 7.7 of [31].

As a technical result for effecting the lifting, we will need to use the stability of the $\gamma$-factor for local generic representations of $\mathrm{SO}_{2 n+1}$ twisted by sufficiently highly ramified characters as in [6]. In the context of the local L-functions defined via Eisenstein series, the existence of the local $\gamma$-factors at all places $v$ is given in Theorem 3.5 of [31] and is related to the local L- and $\varepsilon$-factors by

$$
\gamma\left(s, \pi_{v} \times \tau_{v}, \psi_{v}\right)=\frac{\mathrm{L}\left(1-s, \tilde{\pi}_{v} \times \tilde{\tau}_{v}\right) \varepsilon\left(s, \pi_{v} \times \tau_{v}, \psi_{v}\right)}{\mathrm{L}\left(s, \pi_{v} \times \tau_{v}\right)} .
$$

Proposition 3.4. - (Stability of $\gamma$ ) Let $v$ be a non-archimedean place of $k$ and let $\pi_{1, v}$ and $\pi_{2, v}$ be generic representations of $\mathrm{G}_{n}\left(k_{v}\right)$. Then for every sufficiently highly ramified character $\eta_{v}$ of $k_{v}^{\times}$we have

$$
\gamma\left(s, \pi_{1, v} \times \eta_{v}, \psi_{v}\right)=\gamma\left(s, \pi_{2, v} \times \eta_{v}, \psi_{v}\right)
$$

i.e, after a sufficiently highly ramified twist all $\boldsymbol{\gamma}$-factors become stable.

Proof. - This result was proven in [6] in the context of $\gamma$-factors as defined via the integral representations of L-functions for $\mathrm{SO}_{2 n+1}$ as developed by Gelbart and Piatetski-Shapiro [9], Ginzburg [11], and Soudry [34-36]. However, by Corollary 2 of [35] we know that the two definitions of the $\gamma$-factor agree, at least up to a constant of absolute value one which, in our case, depends only on the character. Hence the result transfers to the case under consideration. 
To determine this stable form, it suffices to take $\pi_{v}$ to be a full induced representation from characters $\mu_{1, v}, \ldots, \mu_{n, v}$ of $k_{v}^{\times}$. Then, since the $\gamma$-factors are inductive, again by Theorem 3.5 of [31], we see that for sufficiently highly ramified $\eta_{v}$ we have

$$
\gamma\left(s, \pi_{v} \times \eta_{v}, \psi_{v}\right)=\prod_{j=1}^{n} \gamma\left(s, \mu_{j, v} \eta_{v}, \psi_{v}\right) \gamma\left(s, \mu_{j, v}^{-1} \eta_{v}, \psi_{v}\right) .
$$

For our purposes it will be better to have the corresponding statement in terms of the L-factors and $\varepsilon$-factors. We begin with $\mathrm{L}$.

Lemma 3.4. - Let $v$ be a non-archimedean place of $k$ and let $\pi_{v}$ be a generic representation of $\mathrm{G}_{n}\left(k_{v}\right)$. Then for every sufficiently highly ramified character $\eta_{v}$ of $k_{v}^{\times}$we have $\mathrm{L}\left(s, \pi_{v} \times \eta_{v}\right) \equiv 1$.

Proof. - This follows from the Main Lemma 1 of [33].

If we combine the stable form of the $\gamma$-factor in (3.2), the relation of the $\gamma$-factor to the L-factor and the $\varepsilon$-factor in (3.1), and this Lemma, we arrive at the following corollary of Proposition 3.4.

Corollary. - Let $v$ be a non-archimedean place of $k$ and let $\pi_{v}$ be a generic representation of $\mathrm{G}_{n}\left(k_{v}\right)$. Let $\mu_{1, v}, \ldots, \mu_{n, v}$ be $n$ characters of $k_{v}^{\times}$. Then for every sufficiently highly ramified character $\eta_{v}$ of $k_{v}^{\times}$we have

$$
\varepsilon\left(s, \pi_{v} \times \eta_{v}, \psi_{v}\right)=\prod_{j=1}^{n} \varepsilon\left(s, \mu_{j, v} \eta_{v}, \psi_{v}\right) \varepsilon\left(s, \mu_{j, v}^{-1} \eta_{v}, \psi_{v}\right)
$$

and

$$
\mathrm{L}\left(s, \pi_{v} \times \eta_{v}\right) \equiv 1
$$

\section{The Local Liftings}

In this section we describe the local liftings in more detail. There are three distinct cases. Let $\pi=\otimes^{\prime} \pi_{v}$ be a globally generic irreducible cuspidal automorphic representation of $\mathrm{SO}_{2 n+1}(\mathbf{A})$.

(i) The non-archimedean unramified lifting

Let $v$ be a place where $\pi_{v}$ is unramified. Then, by the Satake isomorphism $[29,2], \pi_{v}$ is determined by a semi-simple conjugacy class $\left[t_{v}\right]$ in $\operatorname{Sp}_{2 n}(\mathbf{C})$ and the local Langlands L-function is defined by

$$
\mathrm{L}\left(s, \pi_{v}\right)=\operatorname{det}\left(1-t_{v} q_{v}^{-s}\right)^{-1}
$$


where $q_{v}$ is the order of the residue field of $k_{v}$. Under the natural embedding $\imath: \mathrm{Sp}_{2 n}(\mathbf{G}) \hookrightarrow \mathrm{GL}_{2 n}(\mathbf{G}),\left[t_{v}\right]$ determines a semi-simple conjugacy class $\left[\imath\left(t_{v}\right)\right]$ in $\mathrm{GL}_{2 n}(\mathbf{G})$ and this has associated to it an unramified representation $\Pi_{v}$ of $\mathrm{GL}_{2 n}\left(k_{v}\right)$. $\Pi_{v}$ is the local Langlands lift of $\pi_{v}$ to $\mathrm{GL}_{2 n}\left(k_{v}\right)$.

Proposition 4.1. - Let $k_{v}$ be a non-archimedean local field. Then the local Langlands lift $\Pi_{v}$ of an irreducible generic unramified unitary representation $\pi_{v}$ of $\mathrm{SO}_{2 n+1}\left(k_{v}\right)$ to $\mathrm{GL}_{2 n}\left(k_{v}\right)$ is again irreducible, generic, unramified, and self-contragredient.

Proof. — The lift $\Pi_{v}$ is unramified and irreducible by construction.

That the lift is self-contragredient is a simple argument on Satake parameters. Suppose that $\pi_{v}$ is the unramified constituent of the unramified principal series $\operatorname{Ind}_{\mathrm{B}_{n}\left(k_{v}\right)}^{\mathrm{G}_{n}\left(k_{v}\right)}\left(\mu_{1, v} \otimes \ldots \otimes \mu_{n, v}\right)$ with the $\mu_{i, v}$ unramified characters. Then the Satake parameter of $\pi_{v}$ is represented by the diagonal element

$$
t_{v}=\operatorname{diag}\left(\mu_{1, v}\left(\varpi_{v}\right), \ldots, \mu_{n, v}\left(\varpi_{v}\right), \mu_{n, v}\left(\varpi_{v}\right)^{-1}, \ldots, \mu_{1, v}\left(\varpi_{v}\right)^{-1}\right) .
$$

in $\mathrm{Sp}_{2 n}(\mathbf{G})$, where $\boldsymbol{\varpi}_{v}$ is a uniformizing parameter for $k_{v}$. Under the embedding $\imath: \mathrm{Sp}_{2 n}(\mathbf{G}) \hookrightarrow \mathrm{GL}_{2 n}(\mathbf{G})$ we have $\mathbf{\imath}\left(t_{v}\right)=t_{v}$ and $\Pi_{v}$ is the unramified representation of $\mathrm{GL}_{2 n}\left(k_{v}\right)$ associated to $\left[t_{v}\right] \subset \mathrm{GL}_{2 n}(\mathbf{G})$. The contragredient representation $\widetilde{\Pi}_{v}$ is then the unramified representation associated to the semi-simple conjugacy class $\left[t_{v}^{-1}\right] \subset \mathrm{GL}_{2 n}(\mathbf{G})$. In $\mathrm{GL}_{2 n}(\mathbf{G})$ we have that $t_{v}$ and $t_{v}^{-1}$ are conjugate by the long Weyl element. Hence $\widetilde{\Pi}_{v} \simeq \Pi_{v}$ as claimed.

It remains to show that $\Pi_{v}$ is generic. We will give two proofs of this fact, in the hope that one might eventually extend to other classical groups.

The first is based on the Kazhdan-Lusztig parameterization of representations having an Iwahori fixed vector [25] and is independent of the unitarity assumption. If $\mathrm{G}$ is any split reductive group over $k_{v}$ with connected center, such as our $\mathrm{SO}_{2 n+1}\left(k_{v}\right)$ or $\mathrm{GL}_{2 n}\left(k_{v}\right)$, then by an independent result of Barbasch-Moy [1], Li [24], or Reeder [28] any irreducible generic unramified representation $\rho$ must be a full unramified principal series induced off the Borel subgroup. If $\rho$ corresponds to a semi-simple conjugacy class $[s]$ in ${ }^{\mathrm{L}} \mathrm{G}^{0}$ then the associated induced representation from the Borel reduces into constituents $\rho(s, u, \chi)$ parameterized by the unipotent elements $u$ of ${ }^{\mathrm{L}} \mathrm{G}^{0}$ such that $A d(s) u=u^{q_{v}}$ and certain irreducible representations $\chi$ of the component group $\mathrm{A}(s, u)=\operatorname{Cent}(s, u) / \operatorname{Cent}(s, u)^{0} \mathrm{LZ}$ where $\operatorname{Cent}(s, u)$ is the centralizer in ${ }^{\mathrm{L}} \mathrm{G}^{0}$ of $s$ and $u$ and $\mathrm{L} Z$ is the center of ${ }^{\mathrm{L}} \mathrm{G}$. The associated induced representation is irreducible iff $s$ supports no non-trivial unipotents in this sense (see [1, 25]). In fact, the proof of Barbasch and Moy cited above shows that if $\rho$ is both generic and unramified it must correspond to a conjugacy class $[s]$ which does not support a non-trivial unipotent and is hence irreducible. 
Now assume that $\pi_{v}$ corresponds to the semi-simple conjugacy class $\left[t_{v}\right]$ in $\operatorname{Sp}_{2 n}(\mathbf{C})$ as above. Since $\pi_{v}$ is generic and unramified, $t_{v}$ cannot support a non-trivial unipotent in $\mathrm{Sp}_{2 n}(\mathbf{G})$. Under the embedding $\mathfrak{\imath}: \mathrm{Sp}_{2 n}(\mathbf{G}) \hookrightarrow \mathrm{GL}_{2 n}(\mathbf{G}), \mathfrak{\imath}\left(t_{v}\right)=t_{v}$. Since the image of $\mathrm{Sp}_{2 n}(\mathbf{G})$ in $\mathrm{GL}_{2 n}(\mathbf{G})$ is "big", if $t_{v}$ cannot support a non-trivial unipotent in $\operatorname{Sp}_{2 n}(\mathbf{G})$ it cannot support one in $\mathrm{GL}_{2 n}(\mathbf{G})$ either since the relevant roots of $\mathrm{GL}_{2 n}$ already lie in $\mathrm{Sp}_{2 n}$. Hence $\Pi_{v}$ must also be a full induced from the Borel and hence generic.

The second proof is based on our knowledge of the unitary dual of $\mathrm{SO}_{2 n+1}\left(k_{v}\right)$. By the result of Barbasch-Moy, Li, or Reeder cited above we still have that $\pi_{v}$ is a full unramified principal series. If we write this in Langlands form, then by the work of Yoshida [38] we know that since in addition $\pi_{v}$ is unitary, it must be of the form

$$
\pi_{v} \simeq \operatorname{Ind}_{\mathrm{B}_{n}\left(k_{v}\right)}^{\mathrm{G}_{n}\left(k_{v}\right)}\left(\mu_{1, v}^{\circ} v^{a_{1}} \otimes \ldots \otimes \mu_{n, v}^{\circ} v^{a_{n}}\right)
$$

with the $\mu_{i, v}^{\circ}$ unramified unitary, $v(x)=|x|_{v}$, and $\frac{1}{2}>a_{1} \geqslant \ldots \geqslant a_{n} \geqslant 0$. Then the local lift $\Pi_{v}$ is simply

$$
\Pi_{v} \simeq \operatorname{Ind}_{\mathrm{B}_{2 n}^{\prime}\left(k_{v}\right)}^{\mathrm{GL}_{22}\left(k_{v}\right)}\left(\mu_{1, v}^{\circ} v^{a_{1}} \otimes \ldots \otimes \mu_{n, v}^{\circ} \boldsymbol{v}^{a_{n}} \otimes\left(\mu_{n, v}^{\circ}\right)^{-1} v^{-a_{n}} \otimes \ldots \otimes\left(\mu_{1, v}^{\circ}\right)^{-1} v^{-a_{1}}\right)
$$

where $\mathrm{B}_{2 n}^{\prime}$ is the standard Borel subgroup of $\mathrm{GL}_{2 n}$. This is then irreducible by [39]. Hence $\Pi_{v}$ is generic.

We will need to control the twists of these L-functions by arbitrary generic representations $\tau_{v}$ of $\mathrm{GL}_{r}\left(k_{v}\right)$ with $r<2 n$.

Proposition 4.2. - Let $\pi_{v}$ be an irreducible unramified generic representation of $\mathrm{G}_{n}\left(k_{v}\right)$ and $\tau_{v}$ be an irreducible generic representation of $\mathrm{GL}_{r}\left(k_{v}\right)$ with $r<2 n$. Then

$$
\mathrm{L}\left(s, \pi_{v} \times \tau_{v}\right)=\mathrm{L}\left(s, \Pi_{v} \times \tau_{v}\right)
$$

and

$$
\varepsilon\left(s, \pi_{v} \times \tau_{v}, \psi_{v}\right)=\varepsilon\left(s, \Pi_{v} \times \tau_{v}, \psi_{v}\right) .
$$

Proof. - As in the proof of Proposition 4.1 we may write

$$
\pi_{v} \simeq \operatorname{Ind}_{\mathrm{B}_{n}\left(k_{v}\right)}^{\mathrm{G}_{n}\left(k_{v}\right)}\left(\mu_{1, v} \otimes \ldots \otimes \mu_{n, v}\right)
$$

and

$$
\Pi_{v} \simeq \operatorname{Ind}_{\mathrm{B}_{2 n}^{\prime}\left(k_{v}\right)}^{\mathrm{GL}_{2 n}\left(k_{v}\right)}\left(\mu_{1, v} \otimes \ldots \otimes \mu_{n, v} \otimes \mu_{n, v}^{-1} \otimes \ldots \otimes \mu_{1, v}^{-1}\right) .
$$


By Theorem 3.1 of [13] we know that

$$
\gamma\left(s, \Pi_{v} \times \tau_{v}, \psi_{v}\right)=\prod_{i=1}^{n} \gamma\left(s, \tau_{v} \otimes \mu_{i, v}, \psi_{v}\right) \gamma\left(s, \tau_{v} \otimes \mu_{i, v}^{-1}, \psi_{v}\right)
$$

and by Theorem 9.5 of the same paper that

$$
\mathrm{L}\left(s, \Pi_{v} \times \tau_{v}\right)=\prod_{i=1}^{n} \mathrm{~L}\left(s, \tau_{v} \otimes \mu_{i, v}\right) \mathrm{L}\left(s, \tau_{v} \otimes \mu_{i, v}^{-1}\right) .
$$

Since Theorem 9.5 applies equally well to the contragredients, then together these statements give also

$$
\varepsilon\left(s, \Pi_{v} \times \tau_{v}, \psi_{v}\right)=\prod_{i=1}^{n} \varepsilon\left(s, \tau_{v} \otimes \mu_{i, v}, \psi_{v}\right) \varepsilon\left(s, \tau_{v} \otimes \mu_{i, v}^{-1}, \psi_{v}\right) .
$$

For the representation $\pi_{v}$ of $\mathrm{G}_{n}\left(k_{v}\right)$ the same results can be extracted from the results of [31, 32]. First, by either the inductivity of $\gamma$ from Theorem 3.5 of [31] or Section 4 of [32] we know

$$
\gamma\left(s, \pi_{v} \times \tau_{v}, \psi_{v}\right)=\prod_{i=1}^{n} \gamma\left(s, \tau_{v} \otimes \mu_{i, v}, \psi_{v}\right) \gamma\left(s, \tau_{v} \otimes \mu_{i, v}^{-1}, \psi_{v}\right) .
$$

To obtain the factorization of the L-factors, first note that since both $\pi_{v}$ and $\tau_{v}$ are generic, they are both full induced from generic tempered representations in Langlands order, i.e.,

$$
\pi_{v} \simeq \operatorname{Ind}_{\mathrm{Q}\left(k_{v}\right)}^{\mathrm{G}_{n}\left(k_{v}\right)}\left(\pi_{1, v}^{\prime} v^{a_{1}} \otimes \ldots \otimes \pi_{m, v}^{\prime} v^{a_{m}} \otimes \pi_{v}^{\prime \prime}\right)
$$

with each $\pi_{i, v}^{\prime}$ tempered on some $\mathrm{GL}_{n_{i}}\left(k_{v}\right), \boldsymbol{v}$ the character $\boldsymbol{v}(g)=|\operatorname{det}(g)|_{v}$ for $g \in \mathrm{GL}_{n_{i}}\left(k_{v}\right)$, $\pi_{v}^{\prime \prime}$ tempered on $\mathrm{G}_{l}\left(k_{v}\right), a_{1}>\ldots>a_{m}$, and $\mathrm{Q}$ the standard parabolic with Levi of the form $\mathrm{GL}_{n_{1}} \times \ldots \times \mathrm{GL}_{n_{m}} \times \mathrm{G}_{l}$. Note that under our assumptions, each $\pi_{i, v}^{\prime}$ and $\pi_{v}^{\prime \prime}$ are full induced from unitary characters. Similarly

$$
\tau_{v} \simeq \operatorname{Ind}_{Q^{\prime}\left(k_{v}\right)}^{\mathrm{GL}_{r}\left(k_{v}\right)}\left(\tau_{1, v}^{\prime} v^{b_{1}} \otimes \ldots \otimes \tau_{t, v}^{\prime} v^{b_{t}}\right)
$$

with each $\tau_{j, v}^{\prime}$ tempered on some $\mathrm{GL}_{r_{j}}\left(k_{v}\right)$ and $\mathrm{Q}^{\prime}$ the standard parabolic with Levi $\mathrm{GL}_{r_{1}} \times \ldots \times \mathrm{GL}_{r_{t}}$. For $\mathrm{G}_{n}\left(k_{v}\right)$ this is the work of Muic [27] and for $\mathrm{GL}_{r}\left(k_{v}\right)$ that of Jacquet and Shalika [16] or Zelevinsky [39]. For such representations, the L-function is defined via this parameterization in Section 7 of [31] as

$$
\mathrm{L}\left(s, \pi_{v} \times \tau_{v}\right)=\prod_{i, j} \mathrm{~L}\left(s+a_{i}+b_{j}, \pi_{i, v}^{\prime} \times \tau_{j, v}^{\prime}\right) \mathrm{L}\left(s-a_{i}+b_{j}, \tilde{\pi}_{i, v}^{\prime} \times \tau_{j, v}^{\prime}\right) \prod_{j} \mathrm{~L}\left(s+b_{j}, \pi_{v}^{\prime \prime} \times \tau_{j, v}^{\prime}\right) .
$$


Consider one of the factors on the right, say $\mathrm{L}\left(s+a_{i}+b_{j}, \pi_{i, v}^{\prime} \times \tau_{j, v}^{\prime}\right)$. Since as we noted $\pi_{i, v}^{\prime}$ is a full induced from unitary characters, say $\pi_{i, v}^{\prime} \simeq \operatorname{Ind}\left(\mu_{1, v}^{i} \otimes \ldots \otimes \mu_{n_{i}, v}^{i}\right)$, and the fact that $\tau_{j, v}^{\prime}$ is tempered, then either by the result of Jacquet, Piatetski-Shapiro and Shalika quoted above or Theorem 5.2 of [32] we have

$$
\mathrm{L}\left(s+a_{i}+b_{j}, \pi_{i, v}^{\prime} \times \tau_{j, v}^{\prime}\right)=\prod_{\ell} \mathrm{L}\left(s+a_{i}+b_{j}, \mu_{\ell, v}^{i} \otimes \tau_{j, v}^{\prime}\right) .
$$

Note again that the Conjecture 5.1, which is a hypothesis of Theorem 5.2 of [32], is known in our case by Theorem 4.1 of [3]. The result of [32] applies equally well to the factor $\mathrm{L}\left(s+b_{j}, \pi_{v}^{\prime \prime} \times \tau_{j, v}^{\prime}\right)$ and hence we may factor $\pi_{v}$ all the way down to its inducing characters $\mu_{1, v}, \ldots, \mu_{n, v}$. If we now reconstruct these decompositions we arrive at

$$
\mathrm{L}\left(s, \pi_{v} \times \tau_{v}\right)=\prod_{i=1}^{n} \mathrm{~L}\left(s, \tau_{v} \otimes \mu_{i, v}\right) \mathrm{L}\left(s, \tau_{v} \otimes \mu_{i, v}^{-1}\right)
$$

and hence, as above,

$$
\varepsilon\left(s, \pi_{v} \times \tau_{v}, \psi_{v}\right)=\prod_{i=1}^{n} \varepsilon\left(s, \tau_{v} \otimes \mu_{i, v}, \psi_{v}\right) \varepsilon\left(s, \tau_{v} \otimes \mu_{i, v}^{-1}, \psi_{v}\right) .
$$

Comparing these factorizations then gives the statements of the Proposition.

\section{(ii) The archimedean lift}

Let $v \mid \infty$ denote an archimedean place. By the arithmetic Langlands classification $[23,2], \pi_{v}$ is parameterized by an admissible homomorphism $\boldsymbol{\varphi}_{v}: \mathrm{W}_{v} \rightarrow \operatorname{Sp}_{2 n}(\mathbf{C})$ where $\mathrm{W}_{v}$ is the Weil group of $k_{v}$. By composing with $1: \mathrm{Sp}_{2 n}(\mathbf{G}) \hookrightarrow \mathrm{GL}_{2 n}(\mathbf{G})$ we have an admissible homomorphism $\mathfrak{\imath} \circ \varphi_{v}: \mathrm{W}_{v} \longrightarrow \mathrm{GL}_{2 n}(\mathbf{G})$ and this defines an irreducible admissible representation $\Pi_{v}$ of $\mathrm{GL}_{2 n}\left(k_{v}\right)$. We take $\Pi_{v}$ as the local lift of $\pi_{v}$.

The local archimedean L- and $\varepsilon$-factors defined via the theory of Eisenstein series we are using are the same as the Artin factors defined through the arithmetic Langlands classification [30]. Since the embedding $\mathbf{l}: \mathrm{Sp}_{2 n}(\mathbf{G}) \hookrightarrow \mathrm{GL}_{2 n}(\mathbf{G})$ is the standard representation of the L-group of $\mathrm{SO}_{2 n+1}\left(k_{v}\right)$ then by the definition of the local L- and $\varepsilon$-factors given in [2] we have

$$
\mathrm{L}\left(s, \boldsymbol{\pi}_{v}\right)=L\left(s, \imath \circ \varphi_{v}\right)=L\left(s, \Pi_{v}\right)
$$

and

$$
\varepsilon\left(s, \pi_{v}, \psi_{v}\right)=\varepsilon\left(s, \imath \circ \varphi_{v}, \psi_{v}\right)=\varepsilon\left(s, \Pi_{v}, \psi_{v}\right)
$$

where in both instances the middle factor is the local Artin-Weil L- and $\varepsilon$-factor attached to representations of the Weil group as in [37].

If $\tau_{v}$ is an irreducible admissible representation of $\mathrm{GL}_{r}\left(k_{v}\right)$ then it is in turn parameterized by an admissible homomorphism $\varphi_{v}^{\prime}: \mathrm{W}_{v} \longrightarrow \mathrm{GL}_{r}(\mathbf{G})$. Then the tensor 
product $\left(\boldsymbol{1} \circ \varphi_{v}\right) \otimes \varphi_{v}^{\prime}: \mathrm{W}_{v} \longrightarrow \mathrm{GL}_{2 n r}(\mathbf{G})$ is admissible and again we have by definition

$$
\mathrm{L}\left(s, \pi_{v} \times \tau_{v}\right)=\mathrm{L}\left(s,\left(\boldsymbol{l} \circ \varphi_{v}\right) \otimes \varphi_{v}^{\prime}\right)=\mathrm{L}\left(s, \Pi_{v} \times \tau_{v}\right)
$$

and

$$
\varepsilon\left(s, \pi_{v} \times \tau_{v}, \psi_{v}\right)=\varepsilon\left(s,\left(1 \circ \varphi_{v}\right) \otimes \varphi_{v}^{\prime}, \psi_{v}\right)=\varepsilon\left(s, \Pi_{v} \times \tau_{v}, \psi_{v}\right) .
$$

Hence at the archimedean places we also have the analogue of Proposition 4.2.

Proposition 4.3. - Let $\pi_{v}$ be an irreducible admissible generic representation of $\mathrm{G}_{n}\left(k_{v}\right), \Pi_{v}$ its local lift to $\mathrm{GL}_{2 n}\left(k_{v}\right)$, and $\tau_{v}$ an irreducible admissible generic representation of $\mathrm{GL}_{r}\left(k_{v}\right)$ with $r<2 n$. Then

$$
\mathrm{L}\left(s, \pi_{v} \times \tau_{v}\right)=\mathrm{L}\left(s, \Pi_{v} \times \tau_{v}\right)
$$

and

$$
\varepsilon\left(s, \pi_{v} \times \tau_{v}, \psi_{v}\right)=\varepsilon\left(s, \Pi_{v} \times \tau_{v}, \psi_{v}\right) .
$$

(iii) The ramified non-archimedean local lift

If $v$ is a non-archimedean place where $\pi_{v}$ is ramified, we will take for $\Pi_{v}$ any irreducible admissible representation of $\mathrm{GL}_{2 n}\left(k_{v}\right)$ which has trivial central character. If we like we may take $\Pi_{v}$ unramified, generic, self-contragredient...

Proposition 4.4. - Let $v \in \mathrm{S}$ and let $\tau_{v}$ be a local component of a cuspidal representation $\tau$ in $\mathscr{T}(\mathrm{S} ; \eta)$. Then as long as $\eta_{v}$ is sufficiently ramified at $v$, with the necessary degree of ramification depending only on $\pi_{v}$ and $\Pi_{v}$, we have

$$
\mathrm{L}\left(s, \pi_{v} \times \tau_{v}\right)=\mathrm{L}\left(s, \Pi_{v} \times \tau_{v}\right)
$$

and

$$
\varepsilon\left(s, \pi_{v} \times \tau_{v}, \psi_{v}\right)=\varepsilon\left(s, \Pi_{v} \times \tau_{v}, \psi_{v}\right)
$$

Proof. - The proof of this Proposition is essentially the same as that of Proposition 4.2. In this case, by the definition of $\mathscr{T}(\mathrm{S} ; \eta)$ we know that $\tau$ can be written as

$$
\tau \simeq \operatorname{Ind}_{\mathrm{B}_{r}^{\prime}\left(k_{v}\right)}^{\mathrm{GL}_{v}\left(k_{v}\right)}\left(\chi_{1, v} \otimes \ldots \otimes \chi_{r, v}\right) \otimes \eta_{v}
$$

with each $\chi_{i, v}$ unramified. If we let $\chi_{i, v}(x)=|x|_{v}^{b_{i}}$ and let $v(x)=|x|_{v}$ then we may write this as

$$
\tau \simeq \operatorname{Ind}_{\mathrm{B}_{r}^{\prime}\left(k_{v}\right)}^{\mathrm{GL}_{r}\left(k_{v}\right)}\left(\eta_{v} v^{b_{1}} \otimes \ldots \otimes \eta_{v} v^{b_{r}}\right)
$$


Arguing as in the proof of Proposition 4.2, but now factoring $\tau$ according to its characters, we find

$$
\mathrm{L}\left(s, \pi_{v} \times \tau_{v}\right)=\prod_{i=1}^{r} \mathrm{~L}\left(s+b_{i}, \pi_{v} \times \eta_{v}\right)
$$

and

$$
\varepsilon\left(s, \pi_{v} \times \tau_{v}, \psi_{v}\right)=\prod_{i=1}^{r} \varepsilon\left(s+b_{i}, \pi_{v} \times \eta_{v}, \psi_{v}\right) .
$$

By the Corollary to Proposition 3.4, we have that for $\eta_{v}$ sufficiently ramified (depending on $\left.\pi_{v}\right) \mathrm{L}\left(s, \pi_{v} \times \tau_{v}\right)=1$ and that each $\varepsilon$-factor becomes standard as stated there. have

On the other hand, by the same results of [13] as used in Proposition 4.2, we

$$
\mathrm{L}\left(s, \Pi_{v} \times \tau_{v}\right)=\prod_{i=1}^{r} \mathrm{~L}\left(s+b_{i}, \Pi_{v} \times \eta_{v}\right)
$$

and

$$
\varepsilon\left(s, \Pi_{v} \times \tau_{v}, \psi_{v}\right)=\prod_{i=1}^{r} \varepsilon\left(s+b_{i}, \Pi_{v} \times \eta_{v}, \psi_{v}\right) .
$$

Now, by the results of Jacquet and Shalika on the stability of L- and $\varepsilon$-factors for $\mathrm{GL}_{n}$ [16] we have that for sufficiently ramified $\eta_{v}$ (depending on $\left.\Pi_{v}\right) \mathrm{L}\left(s, \Pi_{v} \times \tau_{v}\right)=1$ and each $\varepsilon$-factor becomes standard as stated in Proposition 2.2 of [16]. Since the central character of $\Pi_{v}$ is trivial, we see that the stable forms in Proposition 2.2 of [16] and in the Corollary to Proposition 3.4 above agree. This gives the statement of the Proposition.

\section{The Global Lift}

In this section we prove the Theorem stated in Section 1.

Proof. - We begin with $\pi=\otimes^{\prime} \pi_{v}$ an irreducible globally generic cuspidal automorphic representation of $\mathrm{SO}_{2 n+1}(\mathbf{A})$. To each local component $\pi_{v}$ we have associated a local lift $\Pi_{v}$ on $\mathrm{GL}_{2 n}\left(k_{v}\right)$ in Section 4. Let $\Pi=\otimes^{\prime} \Pi_{v}$. Then $\Pi$ is an irreducible admissible representation of $\mathrm{GL}_{2 n}(\mathbf{A})$ with trivial central character. Let $\mathrm{S}$ be a non-empty finite set of finite places so that $\pi_{v}$ is unramified for all finite $v \notin \mathrm{S}$. Then by Propositions 4.2 and 4.3 we have $\mathrm{L}^{\mathrm{S}}(s, \pi)=\mathrm{L}^{\mathrm{S}}(s, \Pi)$ so that the completed L-function $\mathrm{L}(s, \Pi)$ is absolutely convergent in some right half plane. 
Take $\eta=\otimes \eta_{v}$ a unitary character of $k^{\times} \backslash \mathbf{A}^{\times}$such that for $v \in \mathrm{S}$ the local component $\eta_{v}$ is sufficiently ramified so that Proposition 4.4 holds and such that at one place $\eta_{v}^{2}$ is still ramified.

Let $\tau \in \mathscr{T}(\mathrm{S} ; \eta)$. Then by Propositions $4.2-4.4$ we have

$$
\begin{aligned}
\mathrm{L}\left(s, \Pi_{v} \times \tau_{v}\right) & =\mathrm{L}\left(s, \pi_{v} \times \tau_{v}\right) \\
\varepsilon\left(s, \Pi_{v} \times \tau_{v}, \psi_{v}\right) & =\varepsilon\left(s, \pi_{v} \times \tau_{v}, \psi_{v}\right)
\end{aligned}
$$

for all places $v$ of $k$ and hence globally

$$
\begin{aligned}
\mathrm{L}(s, \Pi \times \tau) & =\mathrm{L}(s, \pi \times \tau) \\
\varepsilon(s, \Pi \times \tau) & =\varepsilon(s, \pi \times \tau) .
\end{aligned}
$$

We of course have the analogous statements for the contragredients.

By Propositions 3.1 and 3.2 we know that, for our choice of $\eta, L(s, \Pi \times \tau)$ is entire and bounded in vertical strips. Furthermore, from Proposition 3.3 we have the functional equation

$$
\mathrm{L}(s, \pi \times \tau)=\varepsilon(s, \pi \times \tau) \mathrm{L}(1-s, \tilde{\pi} \times \tilde{\tau})
$$

and hence

$$
\mathrm{L}(s, \Pi \times \tau)=\varepsilon(s, \Pi \times \tau) \mathrm{L}(1-s, \widetilde{\Pi} \times \widetilde{\tau})
$$

Our representation $\Pi$ now satisfies the hypotheses of the Converse Theorem of Section 2. Hence there is an irreducible automorphic representation $\Pi^{\prime}$ such that $\Pi_{v}^{\prime} \simeq \Pi_{v}$ for all $v \notin \mathrm{S}$. In particular, at almost all places $v$ where $\pi_{v}$ is unramified (we must exclude any such places in the finite set S) and all archimedean places $v / \infty$ we have $\Pi_{v}^{\prime} \simeq \Pi_{v}$ is the local Langlands lift of $\pi_{v}$. Hence $\Pi^{\prime}$ is a weak global Langlands lift of $\pi$. This proves the Theorem.

\section{Complements}

In this section we would like to give some consequences of our main theorem. Our first three corollaries are global and deal with the questions of uniqueness, selfcontragredience, and genericity of our lifts. The second two are local in nature and show the existence of a unique generic local lift of any generic supercuspidal representation of $\mathrm{SO}_{2 n+1}\left(k_{v}\right)$.

Let $\pi$ be a globally generic cuspidal representation of $\mathrm{SO}_{2 n+1}(\mathbf{A})$. Let $\Pi$ be any weak lift of $\pi$ to $\mathrm{GL}_{2 n}(\mathbf{A})$. By a result of Langlands [22] we know that there is a partition $\mathbf{n}=\left(n_{1}, \ldots, n_{t}\right)$ of $2 n$ and cuspidal representations $\sigma_{i}$ of $\mathrm{GL}_{n_{i}}(\mathbf{A})$ such that $\Pi$ is a constituent of $\Xi=\operatorname{Ind}_{P_{\mathbf{n}}(\mathbf{A})}^{\mathrm{GL}_{2 n}(\mathbf{A})}\left(\boldsymbol{\sigma}_{1} \otimes \ldots \otimes \boldsymbol{\sigma}_{t}\right)$, where $\mathrm{P}_{\mathbf{n}}$ is the standard parabolic subgroup of $\mathrm{GL}_{2 n}$ with Levi subgroup $\mathrm{GL}_{n_{1}} \times \ldots \times \mathrm{GL}_{n_{t}}$. (As all our inductions in this section 
will be of this form, from a standard parabolic associated to a partition of $2 n$, which will be clear from the context, to $\mathrm{GL}_{2 n}$, we will henceforth omit the groups from the notation for induction.) For those finite places $v$ where $\Pi_{v}$ is unramified we have that $\Pi_{v}$ is the unique unramified constituent of $\Xi_{v}=\operatorname{Ind}\left(\sigma_{1, v} \otimes \ldots \otimes \sigma_{t, v}\right)$.

Now suppose that $\Pi^{\prime}$ is another weak lift of $\pi$. Then to $\Pi^{\prime}$ we also have associated a partition $\left(n_{1}^{\prime}, \ldots, n_{t^{\prime}}^{\prime}\right)$ of $2 n$ and cuspidal representations $\sigma_{i}^{\prime}$ of $\mathrm{GL}_{n_{i}^{\prime}}(\mathbf{A})$ such that $\Pi^{\prime}$ is a constituent of $\Xi^{\prime}=\operatorname{Ind}\left(\sigma_{1}^{\prime} \otimes \ldots \otimes \sigma_{t^{\prime}}^{\prime}\right)$ and for those finite places $v$ where $\Pi_{v}^{\prime}$ is unramified we have that $\Pi_{v}^{\prime}$ is the unique unramified constituent of $\Xi_{v}^{\prime}=\operatorname{Ind}\left(\sigma_{1, v}^{\prime} \otimes \ldots \otimes \sigma_{t^{\prime}, v}^{\prime}\right)$.

By construction, for almost all finite places where $\pi_{v}$ is unramified $\Pi_{v} \simeq \Pi_{v}^{\prime}$ is the local lift of $\pi_{v}$. Thus by the classification result of Jacquet and Shalika (Theorem 4.4 of [14]) we have that $t=t^{\prime}$ and up to permutation $n_{i}=n_{i}^{\prime}$ and $\sigma_{i} \simeq \sigma_{i}^{\prime}$ for $i=1, \ldots, t$. So $\pi$ completely determines the partition $\left(n_{1}, \ldots, n_{t}\right)$ of $2 n$ and cuspidal representations $\sigma_{i}$ of $\mathrm{GL}_{n_{i}}(\mathbf{A})$ as above such that every weak lift of $\pi$ is a constituent of $\Xi=\operatorname{Ind}\left(\sigma_{1} \otimes \ldots \otimes \sigma_{t}\right)$. For definiteness, let us write $\sigma_{i}=\sigma_{i}^{\circ} \nu^{s_{i}}$ where $\sigma_{i}^{\circ}$ is unitary cuspidal and $s_{i} \in \mathbf{R}$ and assume that we have numbered the $\sigma_{i}$ so that $s_{1} \geqslant \ldots \geqslant s_{t}$.

Recall from Proposition 4.1 that at the finite places where $\pi_{v}$ is unramified the local lift $\Pi_{v}$ is unramified and self-contragredient. Hence if we consider $\widetilde{\Xi}=\operatorname{Ind}\left(\widetilde{\sigma}_{1} \otimes\right.$ $\left.\cdots \otimes \widetilde{\sigma}_{t}\right)$ we see that for almost all $v$ such that $\widetilde{\Xi}_{v}$ is unramified the unramified constituent will be $\widetilde{\Pi}_{v}$. Since $\Pi_{v} \simeq \widetilde{\Pi}_{v}$ then again applying the classification theorem of Jacquet and Shalika there is a permutation $p$ of $\{1, \ldots, t\}$ such that $\widetilde{\sigma}_{i} \simeq \sigma_{p(i)}$, that is, as a multiset $\left\{\sigma_{1}, \ldots, \sigma_{t}\right\}$ is self-contragredient. Hence $\Xi$ and $\widetilde{\Xi}$ will have the same constituents.

We say that two irreducible automorphic representations $\Pi$ and $\Pi^{\prime}$ of $\mathrm{GL}_{2 n}(\mathbf{A})$ are nearly equivalent if there is a finite set $\mathrm{T}$ of places of $k$ such that $\Pi_{v} \simeq \Pi_{v}^{\prime}$ for all $v \notin \mathrm{T}$. So if $\Pi$ and $\Pi^{\prime}$ are two weak lifts of $\pi$ then they are nearly equivalent. Moreover we see that any constituent of $\Xi$ is nearly equivalent to any weak lift $\Pi$ of $\pi$ and that in fact the constituents of $\Xi$ make up the full near equivalence class of any weak lift of $\pi$. We will call an irreducible automorphic representation $\Pi$ nearly self-contragredient if $\Pi$ is nearly equivalent to its contragredient $\widetilde{\Pi}$. Since $\widetilde{\Xi}$ and $\Xi$ have the same constituents, we see that any weak lift of $\pi$ is nearly self-contragredient.

Thus we have established the following Corollary to our Theorem.

Corollary 1. - Let $\pi$ be a globally generic cuspidal representation of $\mathrm{SO}_{2 n+1}(\mathbf{A})$. Then $\pi$ completely determines a partition $\left(n_{1}, \ldots, n_{t}\right)$ of $2 n$ and a self-contragredient multiset $\left\{\sigma_{1}, \ldots, \sigma_{t}\right\}$ of cuspidal representations of $\mathrm{GL}_{n_{i}}(\mathbf{A})$ as above such that every weak lift of $\pi$ is a constituent of $\Xi=\operatorname{Ind}\left(\sigma_{1} \otimes \ldots \otimes \sigma_{t}\right)$. Moreover any weak lift $\Pi$ is nearly self-contragredient.

In our case, we know by Proposition 4.1 that the unramified constituents of $\Xi_{v}$ are generic. At any place $v$ we have $\Xi_{v}=\operatorname{Ind}\left(\sigma_{1, v} \otimes \ldots \otimes \sigma_{t, v}\right)$ and since each $\sigma_{i, v}$ is generic each $\Xi_{v}$ will have a unique generic constituent. Hence there is a unique 
irreducible generic automorphic representation $\Sigma$ of $\mathrm{GL}_{2 n}(\mathbf{A})$ which is nearly equivalent to any weak lift $\Pi$ of $\pi$. The representation $\Sigma$ itself may not be a weak lift, because it may not have the requisite component at the archimedean places. However, if $\pi_{v}$ is say tempered at all archimedean places, then the local lift $\Pi_{v}$ of $\pi_{v}$ will still be tempered [23, 2] and hence generic [39]. In this case, $\Sigma_{v}$ will be the local lift of $\pi_{v}$ at archimedean $v$ and hence $\Sigma$ will be a weak lift of $\pi$. We gather these facts together in the following Corollary.

Corollary 2. - Let $\pi$ be a globally generic cuspidal representation of $\mathrm{SO}_{2 n+1}(\mathbf{A})$. Then there is a unique generic automorphic representation $\Sigma$ of $\mathrm{GL}_{2 n}(\mathbf{A})$ such that $\Sigma$ is nearly equivalent to any weak lift of $\pi$. If $\pi$ is tempered at all archimedean places, then $\Sigma$ will be a weak lift of $\pi$.

Note that if we had a way of determining that $\Xi$ was irreducible, for example if we had a cuspidal weak lift, then there would be a unique lift $\Pi$ of $\pi$ and this lift would be self-contragredient and generic. One way of obtaining this is to place extra conditions on $\pi$. Recall from [5] that a cuspidal representation $\pi$ of $\mathrm{SO}_{2 n+1}(\mathbf{A})$ is called weakly Ramanujan if for every $\varepsilon>0$ there is a constant $c_{\varepsilon}>0$ and an infinite sequence of places $\left\{v_{m}\right\}$ with the property that each $\pi_{v_{m}}$ is unramified and its Satake parameters

$$
t_{v_{m}}=\operatorname{diag}\left(\mu_{1, v_{m}}, \ldots, \mu_{n, v_{m}}, \mu_{n, v_{m}}^{-1}, \ldots, \mu_{1, v_{m}}^{-1}\right)
$$

satisfy $c_{\varepsilon}^{-1} q_{v_{m}}^{-\varepsilon}<\left|\mu_{i, v_{m}}\right|<c_{\varepsilon} q_{v_{m}}^{\varepsilon}$. If $\pi$ has one tempered unramified component or if $\pi$ is weakly Ramanujan, then by Theorem 1 or Theorem $1^{\prime}$ of [5] we see that the exponents occurring in $\Xi$ are all zero, i.e., $s_{1}=\ldots=s_{t}=0$, so that $\sigma_{i}=\sigma_{i}^{\circ}$ is unitary cuspidal and $\Xi=\operatorname{Ind}\left(\sigma_{1}^{\circ} \otimes \ldots \otimes \sigma_{t}^{\circ}\right)$. Then $\Xi$ is irreducible.

Corollary 3. - If $\pi$ has one tempered unramified component or is weakly Ramanujan, then $\pi$ has a unique lift $\Pi=\Xi$ and the lift is generic and self-contragredient.

Now let us turn to some local consequences of the existence of a weak lift. The local lift at archimedean places is well understood, as explained above, so let us fix a non-archimedean place $v$. If $\pi_{v}$ is an unramified generic representation of $\operatorname{SO}_{2 n+1}\left(k_{v}\right)$ with local lift $\Pi_{v}$, then by Proposition 4.2 we know that for every supercuspidal representation $\tau_{v}$ of $\mathrm{GL}_{r}\left(k_{v}\right)$ we have

$$
\gamma\left(s, \pi_{v} \times \tau_{v}, \psi_{v}\right)=\gamma\left(s, \Pi_{v} \times \tau_{v}, \psi_{v}\right) .
$$

This relationship persists for any local component of a weak lift.

Corollary 4. - Let $\pi_{v}$ be a local component of a globally generic cuspidal representation $\pi$ of $\mathrm{SO}_{2 n+1}(\mathbf{A})$ and let $\Pi$ be any weak lift of $\pi$ to $\mathrm{GL}_{2 n}(\mathbf{A})$. Then for every supercuspidal representation $\tau_{v}$ of $\mathrm{GL}_{r}\left(k_{v}\right)$ with $1 \leqslant r<2 n$ we have

$$
\gamma\left(s, \pi_{v} \times \tau_{v}, \psi_{v}\right)=\gamma\left(s, \Pi_{v} \times \tau_{v}, \psi_{v}\right) .
$$


Proof. - Let $\tau_{v}$ be as in the statement of the Corollary. Then by Proposition 5.1 of [31] there is a cuspidal representation $\tau^{\prime}$ of $\mathrm{GL}_{r}(\mathbf{A})$ such that at the place $v$ the local component of $\tau^{\prime}$ is the given $\tau_{v}$ and at all other finite places $w \neq v$ we have $\tau_{w}^{\prime}$ is unramified. Let $\mathrm{S}$ be a finite set of finite places such that $\pi_{w}$ is unramified for $v \notin \mathrm{S}$ and let $\mathrm{S}^{\prime}=\mathrm{S}-\{v\}$. Let $\eta$ be a idele class character such that $\eta_{v}$ is trivial and $\eta_{w}$ is sufficiently highly ramified at $w \in \mathrm{S}^{\prime}$ so that

$$
\gamma\left(s, \pi_{w} \times\left(\tau_{w}^{\prime} \otimes \eta_{w}\right), \psi_{w}\right)=\gamma\left(s, \Pi_{w} \times\left(\tau_{w}^{\prime} \otimes \eta_{w}\right), \psi_{w}\right)
$$

as in the proof of Proposition 4.4.

Let $\tau=\tau^{\prime} \otimes \eta$. Note that since $\eta_{v}$ is trivial the local component of $\tau$ at $v$ is still our given $\tau_{v}$. We have the global functional equations

$$
\mathrm{L}(s, \pi \times \tau)=\varepsilon(s, \pi \times \tau) \mathrm{L}(1-s, \tilde{\pi} \times \tilde{\tau})
$$

and

$$
\mathrm{L}(s, \Pi \times \tau)=\varepsilon(s, \Pi \times \tau) \mathrm{L}(1-s, \widetilde{\Pi} \times \widetilde{\tau})
$$

which we can write in the form

$$
\gamma\left(s, \pi_{v} \times \tau_{v}, \psi_{v}\right)=\left(\prod_{w \in \mathrm{S}^{\prime}} \gamma\left(s, \pi_{w} \times \tau_{w}, \psi_{w}\right)^{-1}\right) \frac{\mathrm{L}^{\mathrm{S}}(s, \pi \times \tau)}{\varepsilon^{\mathrm{S}}(s, \pi \times \tau, \psi) \mathrm{L}^{\mathrm{S}}(1-s, \tilde{\pi} \times \tilde{\tau})}
$$

and

$$
\gamma\left(s, \Pi_{v} \times \tau_{v}, \psi_{v}\right)=\left(\prod_{w \in \mathrm{S}^{\prime}} \gamma\left(s, \Pi_{w} \times \tau_{w}, \psi_{w}\right)^{-1}\right) \frac{\mathrm{L}^{\mathrm{S}}(s, \Pi \times \tau)}{\varepsilon^{\mathrm{S}}(s, \Pi \times \tau, \psi) \mathrm{L}^{\mathrm{S}}(1-s, \widetilde{\Pi} \times \widetilde{\tau})} .
$$

By Propositions 4.2 and 4.3 we have that

$$
\frac{\mathrm{L}^{\mathrm{S}}(s, \pi \times \tau)}{\varepsilon^{\mathrm{S}}(s, \pi \times \tau, \psi) \mathrm{L}^{\mathrm{S}}(1-s, \tilde{\pi} \times \widetilde{\tau})}=\frac{\mathrm{L}^{\mathrm{S}}(s, \Pi \times \tau)}{\varepsilon^{\mathrm{S}}(s, \Pi \times \tau, \psi) \mathrm{L}^{\mathrm{S}}(1-s, \widetilde{\Pi} \times \widetilde{\tau})},
$$

while for $w \in S^{\prime}$ we have $\gamma\left(s, \pi_{w} \times \tau_{w}, \psi_{w}\right)=\gamma\left(s, \Pi_{w} \times \tau_{w}, \psi_{w}\right)$ by $(6.1)$. Hence

$$
\gamma\left(s, \pi_{v} \times \tau_{v}, \psi_{v}\right)=\gamma\left(s, \Pi_{v} \times \tau_{v}, \psi_{v}\right) .
$$

This establishes the Corollary.

If we push this line of argument a bit further we find the following.

Corollary 5. - Let $v$ be a non-archimedean place of $k$ and let $\pi_{v}$ be an irreducible admissible generic representation of $\mathrm{SO}_{2 n+1}\left(k_{v}\right)$ which appears as a local component of some globally generic 
cuspidal representation. Then there exists a unique generic representation $\Pi_{v}$ of $\mathrm{GL}_{2 n}\left(k_{v}\right)$ such that for every supercuspidal representation $\tau_{v}$ of $\mathrm{GL}_{r}\left(k_{v}\right)$ with $1 \leqslant r<2 n$ we have

$$
\gamma\left(s, \pi_{v} \times \tau_{v}, \psi_{v}\right)=\gamma\left(s, \Pi_{v} \times \tau_{v}, \psi_{v}\right) .
$$

In particular, this is true for any irreducible generic supercuspidal representation $\pi_{v}$. Moreover, if $\pi_{v}$ is the component at $v$ of a globally generic cuspidal representation $\pi$ and $\Pi^{\prime}$ is any weak lift of $\pi$ such that $\Pi_{v}^{\prime}$ is generic, then $\Pi_{v}^{\prime} \simeq \Pi_{v}$, i.e., there is a unique generic local lift of $\pi_{v}$.

Proof. - Take $\pi_{v}$ as the local component at $v$ of the globally generic cuspidal representation $\pi$. We first show the existence of one such $\Pi_{v}$. If $\pi_{v}$ is unramified, then the statement follows from Proposition 4.1 and Proposition 4.2. In general, by Corollary 1 every weak lift $\Pi^{\prime}$ of $\pi$ is a constituent of $\Xi$. If $v$ is finite and $\pi_{v}$ is ramified, then we may choose the local component $\Pi_{v}$ of our weak lift $\Pi$ to be the generic component of $\Xi_{v}$ without effecting the fact that we have a weak lift. Then the statement about the equality of $\gamma$-factors follows from Corollary 4 .

This shows the existence of such $\Pi_{v}$. The uniqueness follows from the "local converse theorem", that is, a generic admissible irreducible representation of $\mathrm{GL}_{2 n}\left(k_{v}\right)$ is uniquely determined by its $\gamma$-factor with twists by supercuspidal representations of all smaller rank general linear groups, as in the Remark after the Corollary of Theorem 1.1 of Henniart [12].

If $\pi_{v}$ is a generic supercuspidal representation of $\mathrm{SO}_{2 n+1}\left(k_{v}\right)$ then by Proposition 5.1 of [31] it occurs as the local component of a globally generic cuspidal representation of $\mathrm{SO}_{2 n+1}(\mathbf{A})$, hence the above reasoning applies.

The final statement of the Corollary has in fact been shown in the beginning part of the proof since we took for $\pi$ an arbitrary global cuspidal representation of $\mathrm{SO}_{2 n+1}(\mathbf{A})$ with local component $\pi_{v}$ and arrived at the uniquely defined local generic lift $\Pi_{v}$.

\section{REFERENCES}

[1] D. Barbasch and A. Moy, Whittaker models with an Iwahori fixed vector, Contemporary Mathematics 177 (1994), 101-105.

[2] A. Borel, Automorphic L-functions, Proc. Symp. Pure Math. 33, part 2 (1979), 27-61.

[3] W. Gasselman and F. Shahidi, On reducibility of standard modules for generic representations, Ann. Sci. École Norm. Sup. 31 (1998), 561-589.

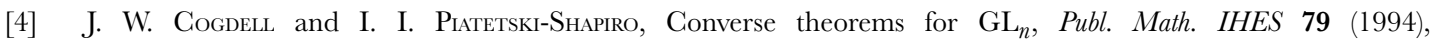
157-214.

[5] J. W. Gogdell and I. I. Piatetski-Shapiro, Unitarity and functoriality, Geom. Funct. Anal. 5 (1995), 164-173.

[6] J. W. Cogdell and I. I. Piatetski-Shapiro, Stability of gamma factors for $\mathrm{SO}(2 n+1)$, Manuscripta math. 95 (1998), 437-461. 
[7] J. W. Cogdell and I. I. Piatetski-Shapiro, Converse theorems for $\mathrm{GL}_{n}$, II, f. reine angew. Math. 507 (1999), 165-188.

[8] J. W. Cogdell and I. I. Piatetski-Shapiro, Converse theorems for $\mathrm{GL}_{n}$ and their application to liftings, to appear in the proceedings of the International Conference on Cohomology of Arithmetic Groups, Automorphic Forms, and L-functions, Tata Institute of Fundamental Research.

[9] S. Gelbart and I. I. Piatetski-Shapiro, L-functions for $\mathrm{G} \times \mathrm{GL}_{n}$, Springer Lecture Notes in Math. 1254, $52-146$.

[10] S. Gelbart and F. Shahidi, Boundedness of automorphic L-functions in finite vertical strips, to appear, fournal of the $A M S$.

[11] D. Ginzburg, L-functions for $\mathrm{SO}_{n} \times \mathrm{GL}_{k}$, f. reine angew. Math. 405 (1990), 156-280.

[12] G. Henniart, Caractérisation de la correspondance de Langlands locale par les facteurs $\varepsilon$ de paires, Invent. Math. 113 (1993), 339-350.

[13] H. Jacquet, I. I. Piatetski-Shapiro and J. Shalika, Rankin-Selberg convolutions, Am. F. Math. 105 (1983), 367-464.

[14] H. Jacquet and J. Shalika, On Euler products and the classification of automorphic representations, I, Am. F. Math. 103 (1981), 499-558; II, 103 (1981), 777-815.

[15] H. Jacquet and J. Shalika, The Whittaker models for induced representations, Pacific F. Math. 109 (1983), 107-120.

[16] H. Jacquet and J. Shalika, A lemma on highly ramified $\varepsilon$-factors, Math. Ann. 271 (1985), 319-332.

[17] H. Jacquet and J. Shalika, Rankin-Selberg Convolutions: Archimedean Theory, Festschrift in Honor of I. I. Piatetski-Shapiro, Part I, Jerusalem, Weizmann Science Press (1990), 125-207.

[18] H. KIM, Langlands-Shahidi method and poles of automorphic L-functions: Applications to exterior square L-functions, Canad. 7. Math. 51 (1999), 835-849.

[19] H. KIM, Langlands-Shahidi method and poles of automorphic L-functions, II, Israel F. Math. 117 (2000), 261-284; Correction, Israel 7. Math. 118 (2000), 379.

[20] R. P. Langlands, Problems in the theory of automorphic forms, Lectures in Modern Analysis and Applications, III, Lecture Notes in Math. 170, Springer Verlag, Berlin (1970), 18-61.

[21] R. P. Langlands, On the Functional Equations Satisfied by Eisenstein Series, Lecture Notes in Mathematics 544, Springer Verlag, Berlin (1976).

[22] R. P. Langlands, On the notion of an automorphic representation, Proc. Sympos. Pure Math. 33, part 1 (1979) 189-207.

[23] R. P. Langlands, On the classification of irreducible representations of real algebraic groups, Representation Theory and Harmonic Analysis on Semisimple Lie Groups, Math. Surveys Monographs, 31, Amer. Math. Soc. Providence, RI (1989), 101-170.

[24] J.-S. LI, Some remarks on unramified principal series of p-adic groups, Math. Ann. 292 (1992), 747-761.

[25] G. Lusztig, Representations of affine Hecke algebras, Astérisque 171-172 (1989), 73-84.

[26] G. Meglin and J.-L. Waldspurger, Spectral decomposition and Eisenstein series. Une paraphrase de l'Écriture, Cambridge Tracts in Mathematics, 113, Cambridge, Cambridge University Press (1995).

[27] G. Muić, Some results on square integrable representations; Irreducibility of standard representations, IMRN No. 14 (1998), 705-726.

[28] M. REeDER, p-adic Whittaker functions and vector bundles on flag manifolds, Compositio Math. 85 (1993), 9-36.

[29] I. Satake, Theory of spherical functions on reductive algebraic groups over p-adic fields, Publ. Math. IHES 18 (1963), 5-69.

[30] F. SHAhidi, Local coefficients as Artin factors for real groups, Duke Math. 7. 52 (1985), 973-1007.

[31] F. SHahidi, A proof of Langlands' conjecture on Plancherel measures; complementary series for $p$-adic groups, Ann. of Math. 132 (1990), 273-330.

[32] F. Shahidi, On multiplicativity of local factors, Festschrift in honor of I.I. Piatetski-Shapiro on the occasion of his sixtieth birthday, Part II (Ramat Aviv, 1989), Israel Math. Conf. Proc., 3, Weizmann, Jerusalem (1990), 279-289. 
[33] F. Shahidi, Twists of a general class of L-functions by highly ramified characters, Canad. Math. Bull. 43 (2000), 380-384.

[34] D. Soudry, Rankin-Selberg convolutions for $\mathrm{SO}_{2 l+1} \times \mathrm{GL}_{n}$; Local theory, Memoirs of the AMS 500 (1993).

[35] D. Soudry, On the Archimedean Theory of Rankin-Selberg convolutions for $\mathrm{SO}_{2 l+1} \times \mathrm{GL}_{n}$, Ann. scient. Éc. Norm. Sup. 28 (1995), 161-224.

[36] D. Soudry, Full multiplicativity of gamma factors for $\mathrm{SO}_{2 l+1} \times \mathrm{GL}_{n}$, preprint.

[37] J. Tate, Number theoretic background, Proc. Symp. Pure Math. 33 (1979), 3-26.

[38] H. Yoshida, On the unitarizability of principal series representations of $\mathfrak{p}$-adic Chevalley groups, f. Math. Kyoto Univ. 32 (1992), 155-233.

[39] A. ZeLevinsky, Induced representations of reductive p-adic groups II, Ann. Sci. École Norm. Sup. 13 (1980), 165-210.

J. W. C.

Department of Mathematics, Oklahoma State University, Stillwater, OK 74078, USA

H. H. K.

Department of Mathematics, Southern Illinois University,

Carbondale, IL 62901, USA

\section{I. P. S.}

Department of Mathematics, Yale University, New Haven, CT 06520, and School of Mathematics,

Tel Aviv University,

Tel Aviv 69978 Israel

F. S.

Department of Mathematics, Purdue University, West Lafayette, IN 47907, USA 